\title{
Detailed abundance study of four s-process enriched post-AGB stars in the Large Magellanic Cloud ${ }^{\star}, \star \star$
}

\author{
E. van Aarle ${ }^{1}$, H. Van Winckel ${ }^{1}$, K. De Smedt ${ }^{1}$, D. Kamath ${ }^{2}$, and P. R. Wood ${ }^{2}$ \\ 1 Instituut voor Sterrenkunde, K.U. Leuven, Celestijnenlaan 200D bus 2401, 3001 Leuven, Belgium \\ e-mail: Hans.VanWinckel@ster . kuleuven.be \\ 2 Research School of Astronomy and Astrophysics, Mount Stromlo Observatory, Weston Creek, ACT 2611, Australia \\ Received 11 July 2012 / Accepted 17 April 2013
}

\section{ABSTRACT}

\begin{abstract}
Context. The photospheric abundances of evolved solar-type stars of different metallicities serve as probes into stellar evolution theory.

Aims. Stellar photospheres of post-asymptotic giant branch (post-AGB) stars bear witness to the internal chemical enrichment processes, integrated over their entire stellar evolution. Here we study post-AGB stars in the Large Magellanic Cloud (LMC). With their known distances, these rare objects are ideal tracers of AGB nucleosynthesis and dredge-up phenomena.

Methods. We used the UVES spectrograph mounted on the Very Large Telescope at the European Southern Observatory, to obtain high-resolution spectra with high signal-to-noise of a sample of four post-AGB stars. The objects display a spectral energy distribution that indicates the presence of circumstellar dust. We perform a detailed abundance analysis on the basis of these spectra.

Results. All objects are C-rich, and strongly enhanced in s-process elements. We deduced abundances of heavy s-process elements for all stars in the sample, and even found an indication of the presence of $\mathrm{Hg}$ in the spectrum of one object. The metallicity of all stars except J053253.51-695915.1 is considerably lower than the average value that is observed for the LMC. The derived luminosities show that we witness the late evolution of low-mass stars with initial masses close to $1 M_{\odot}$. An exception is J053253.51-695915.1 and we argue that this object is likely a binary.

Conclusions. We confirmed the correlation between the efficiency of the third-dredge up and the neutron exposure that is detected in Galactic post-AGB stars. The non-existence of a correlation between metallicity and neutron irradiation is also confirmed and expanded to smaller metallicities. We confirm the status of $21 \mu \mathrm{m}$ stars as post-carbon stars. Current theoretical AGB models overestimate the observed $\mathrm{C} / \mathrm{O}$ ratios and fail to reproduce the variety of s-process abundance patterns that is observed in otherwise very similar objects. Similar results have recently been found for a post-AGB star in the Small Magellanic Cloud (SMC).
\end{abstract}

Key words. stars: AGB and post-AGB - stars: evolution - Magellanic Clouds - circumstellar matter - stars: abundances

\section{Introduction}

Post-asymptotic giant branch (post-AGB) stars are stars of low and intermediate initial mass $\left.(M \lesssim 7) M_{\odot}\right)$ that approach the end of their stellar evolution. The post-AGB evolutionary phase starts when the strong dusty mass loss, that is thought to characterise the end of the AGB, has ceased. The central star will evolve rapidly to the blue of the Hertzsprung-Russell (HR) diagram. Here we concentrate on the photospheric chemical abundances and want to use the photosphere of post-AGB stars as ideal tracers to study AGB nucleosynthetic and dredge-up processes.

Among the known Galactic post-AGB stars, the variety in observed chemical patterns in the photospheres is large (e.g. Van Winckel 2003, and references therein). Some objects are strongly enhanced in neutron capture (s-process) elements, while others are not enhanced at all and might even display s-process deficiencies. This bifurcation is rather strict, as only a limited

* Based on observations collected with the Very Large Telescope at the ESO Paranal Observatory (Chili) of programme number 082.D-0941.

$\star \star$ Detailed line data used for the abundance analysis are only available at the CDS via anonymous ftp to cdsarc.u-strasbg.fr $(130.79 .128 .5)$ or via

http://cdsarc.u-strasbg.fr/viz-bin/qcat?]/A+A/554/A106 number of post-AGB objects are known to be mildly enriched in s-process elements (e.g. Sumangala Rao et al. 2011, and references therein). This large chemical variety and the rather small sample of known Galactic post-AGB stars, makes it hard to understand how these results can be interpret in the framework of chemical AGB evolution models.

One of the problems with the current sample of known Galactic post-AGB stars is that their distances, and hence luminosities and initial masses, are badly determined as practically none of these objects has a reliable parallax measurement. We want to overcome this problem by studying post-AGB objects in other galaxies, and therefore focus in this contribution on the Large Magellanic Cloud (LMC). At a distance of about $50 \mathrm{kpc}$ (Storm et al. 2011) and at a favourable aspect angle of $35^{\circ}$ (van der Marel \& Cioni 2001), the LMC is far enough away that all objects can be assumed to reside at the same distance, and still close enough to allow the observation of individual objects in detail.

Observations of post-AGB stars can help us interpret the AGB phase of which they are the progeny. Studying optically bright post-AGB stars holds several advantages over studying the AGB stars themselves. First, a simultaneous study of the photosphere and the circumstellar environment is possible when the circumstellar envelope becomes optically thin by expansion. Photospheric spectra of AGB stars are veiled by molecular 
Table 1. Names and spectral types of the programme stars (van Aarle et al. 2011).

\begin{tabular}{lc}
\hline \hline $\begin{array}{l}\text { Object name } \\
\text { (IRAC) }\end{array}$ & Spectral type $^{a}$ \\
\hline J050632.10-714229.8 & A3Iab/F0Ibp(e) \\
J052043.86-692341.0 & F5Ib(e) \\
J053250.69-713925.8 & F6Ia \\
J053253.51-695915.1 & G5 \\
\hline
\end{tabular}

Notes. ${ }^{(a)}$ The "p" indicates that more than one low resolution, optical spectrum was obtained.

lines which impedes spectral analysis of trace elements (e.g. Abia et al. 2008). Spectra of post-AGB stars are dominated by atomic transitions rather than molecular transitions and this allows abundance determinations of a wide range of elements. Furthermore, the photospheres of post-AGB stars are convenient to study as the spectral types tend to cluster in a range where model atmosphere analysis in the optical is the most reliable.

We initiated a large project to study quantitatively our newly identified, large sample of post-AGB stars in the LMC (van Aarle et al. 2011) and Small Magellanic Cloud (SMC; Kamath et al. 2011). In this paper, we will present the abundance analysis of four LMC post-AGB stars that were selected from the 70 spectroscopically confirmed post-AGB stars in the catalogue of optically bright post-AGB stars of the LMC (see van Aarle et al. 2011). We introduce the objects in Sect. 2. The high-resolution UVES spectra and their reduction is discussed in Sect. 3. We performed a detailed abundance analysis of the obtained spectra which is presented in Sect. 4. The results of this analysis are discussed in the next sections: in Sect. 6 we use the acquired atmosphere models to constrain the luminosities and initial masses of our program stars, the neutron exposure of the present sample is compared to that of some Galactic post-AGB objects in Sect. 7, Sect. 8 deals with the possibility that one of the objects in the sample is a binary. A first comparison with chemical AGB models is presented in Sect. 9. We end with some conclusions and future prospects in Sect. 10.

\section{The sample stars}

Basic information on four objects is gathered in Table 1. This table lists the full name of each object, which we will abbreviate in the rest of this paper to increase the readability.

Three objects have a clearly double peaked spectral energy distribution (SED; Fig. 7): in which the peak at longer wavelengths is indicative of a freely expanding, detached shell (van Aarle et al. 2011). In the SED of J053253, the fourth object, only a small, relatively hot infrared excess is visible.

All objects in our sample display photometric variability. J052043 and J053250 are semi-regular variables (SRVs) with an unusual warm spectral type, while J050632 and J053253 are population II Cepheids with a very low amplitude and periods of 49.1 and 91.04 days respectively (van Aarle et al. 2011). All objects have a peak-to-peak photometric amplitude of less than $0.1 \mathrm{mag}$.

Only one object in our sample has been studied before. Volk et al. (2011) obtained Spitzer Space Telescope mid-infrared spectroscopy for J052043 and detected a strong $21 \mu \mathrm{m}$ feature. The carrier of this dust feature is still unknown but it is only detected in post-AGB stars (e.g. Hrivnak et al. 2009). For the Galactic sample of post-AGB stars, it is known that the presence
Table 2. Observational $\log$.

\begin{tabular}{lccccc}
\hline \hline Object & Date & $\begin{array}{c}\text { Exp. time } \\
(\mathrm{s})\end{array}$ & $\begin{array}{c}S / N \\
\text { Blue }\end{array}$ & $\begin{array}{c}\operatorname{Red}_{\text {low }} \\
\text { Red }\end{array}$ & $\operatorname{Re}_{\text {up }}$ \\
\hline J050632 & $4-5 / 11 / 2008$ & $2 \times 3005$ & 50 & 105 & 110 \\
J052043 & $21-22 / 11 / 2008$ & $1 \times 3005$ & 10 & 50 & 60 \\
& $4-5 / 12 / 2008^{a}$ & $2 \times 3005$ & 15 & & \\
& & $3 \times 3005$ & & 80 & 100 \\
J053250 & $17-18 / 11 / 2008$ & $3 \times 3005$ & 15 & 90 & 120 \\
J053253 & $19-20 / 10 / 2008$ & $4 \times 3005$ & 10 & 50 & 70 \\
\hline
\end{tabular}

Notes. We list the names, dates, exposure times and signal-to-noise per pixel of all UVES spectra taken for our sample stars. "Blue" corresponds to the spectral range from $3280-4519 \AA$, "Red low" to 4777-5757 $\AA$, and "Red up" to 5834-6810 $\AA$. (a) The third blue spectrum had to be removed due to an instrumental error.

of this feature accompanies a central star which is enriched in carbon and shows a strong enhancement in s-process elements (Van Winckel \& Reyniers 2000; Reddy et al. 2002; Reyniers 2002; Reyniers et al. 2004). In this paper, we will explore if this remains true for this object in the LMC.

\section{Observations}

We obtained high-resolution, high-signal-to-noise UVES spectra (Dekker et al. 2000) for all stars in our sample. The spectra were taken in service mode in the fall of 2008. The spectrograph splits the light beam from the telescope into two spectral arms which are operated simultaneously. This results in a spectral coverage from 3280 to $4519 \AA$ in the blue arm, and from 4777 to $5757 \AA$, and 5834 to $6810 \AA$ in the red arm. We used a slit width of $0.8^{\prime \prime}$ which translates into $\delta \lambda \sim \lambda / 52000$ in the blue and $\delta \lambda \sim \lambda / 48000$ in the red spectral region ${ }^{1}$. Some details of the observations can be found in Table 2 .

The data reduction was performed with the UVES pipeline (version 4.3.0), which included bias correction, flat-fielding, and background, sky, and cosmic hit correction. Even though the image slicer was used to obtain our spectra, we used optimal instead of average extraction to convert the frames from pixel-pixel to pixel-order space. This produced spectra of similar quality but took better care of the remaining cosmic hits.

Before merging the different spectra, the barycentric correction of the spectra, as well as an intrinsic variability check on the different exposures of each object were performed. All spectra are consistent for all objects, except for J052043. Its spectra obtained in December show a small difference in spectral lines with respect to the ones taken in November. We decided not to merge these spectra, but to treat them separately. We will indicate the spectrum taken in November with $a$, and that from December with $b$. We removed some additional cosmic hits by hand, and merged all consistent spectra, thus retaining two spectra for J052043 and one for all other objects. All spectra were normalised by dividing the spectrum by a spline function defined through interactively identified continuum points. The reciprocal of the standard deviation computed on a few normalised continuum windows was used to express the signal-to-noise ratio $(\mathrm{S} / \mathrm{N})$ of our spectra (Table 2) and some sample spectra are shown in Fig. 1.

The high resolution and the large wavelength coverage of our UVES spectra permit us to determine the radial velocities using

\footnotetext{
1 http://www.eso.org/sci/facilities/paranal/ instruments/uves/inst/
} 


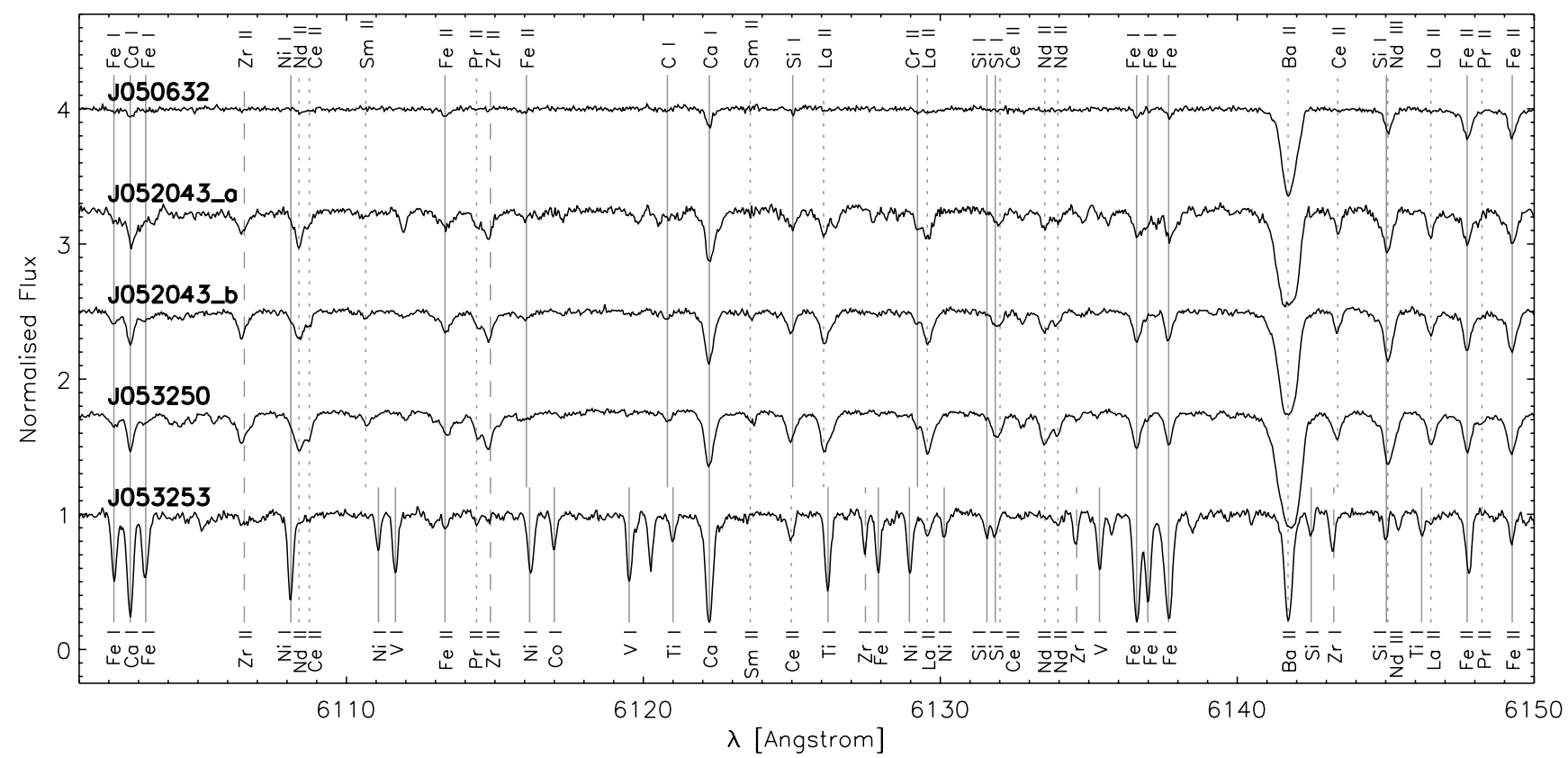

Fig. 1. Example of the quality of the spectra of the stars in our sample. A complete line identification of this spectral interval has been performed using the VALD database (Kupka et al. 1999). Elements with atomic number $Z$ smaller than 30 are indicated with a full line, light s-process elements with a dashed line, and heavy s-process elements with a dotted line. As the lower spectrum differs significantly from the upper four, other features are indicated on it. All objects are clearly enhanced in s-process elements, although this is less obvious in the spectrum of J050632 due to its higher temperature.

Table 3. Heliocentric radial velocities.

\begin{tabular}{lcc}
\hline \hline Object & $\begin{array}{c}v_{r} \\
\left(\mathrm{~km} \mathrm{~s}^{-1}\right)\end{array}$ & $N_{\text {lines }}$ \\
\hline J050632 & $218.2 \pm 0.8$ & 364 \\
J052043_a & $243.3 \pm 1.1$ & 346 \\
J052043_b & $244.6 \pm 1.0$ & 438 \\
J053250 & $283.3 \pm 0.7$ & 278 \\
J053253 & $237.5 \pm 0.8$ & 454 \\
\hline
\end{tabular}

Notes. We list the names, radial velocities and the number of lines used to determine these velocities. The errors on the radial velocity are the standard deviations of the individual results of all lines.

the position of a large number of lines. The line positions were measured using a Gaussian fit through the measured profiles. The identification of the lines and their rest wavelength were taken from the VALD (Kupka et al. 1999) database. The line identification itself was performed by comparing the expected line strength of all input lines using the model atmosphere and overabundances (Sect. 4) of our chemical analyses. We took automatically the nearest strongest line. The internal consistancy on a large number of lines shows that this method is reliable. The results are listed in Table 3 . The radial velocities we find are consistent with the average radial velocity of the LMC, which is about $262 \mathrm{~km} \mathrm{~s}^{-1}$ (van der Marel et al. 2002).

\section{Spectral analyses}

We use the list of lines that are useful for the chemical analysis of A, F, and G stars that has been gathered at the Instituut voor Sterrenkunde and is regularly updated. The initial list is described in Van Winckel \& Reyniers (2000), and contains lines for a range of elements from $\mathrm{He}(Z=2)$ up to $\mathrm{Eu}(Z=63)$. With this list, we ensure that the different chemical analyses are performed homogeneously and we restrict ourselves to lines with reliable oscillator strengths. We determined the equivalent widths of the lines by fitting Gaussian profiles to the observations to take care of blending in their wings.

To determine the atmosphere parameters and obtain the abundances of trace elements, we used the ATLAS 9 model atmospheres (Castelli \& Kurucz 2004) in combination with the latest version (August 2010) of the LTE abundance calculation routine MOOG (Sneden 1973). The model gridsteps are $\Delta T_{\text {eff }}=+/-250 \mathrm{~K}, \Delta \log g=+/-0.5$ and $\Delta([\mathrm{Fe} / \mathrm{H}])=+/-0.5$.

\subsection{Atmospheric parameters}

We used several spectroscopic tracers to quantify the effective temperature of our low gravity objects. A useful upper limit for the effective temperature is provided by the absence of He lines in the spectra. At the $\mathrm{S} / \mathrm{N}$ of our spectra, this means that the lines must be weaker than $\sim 5 \mathrm{~m} \AA$. The absence of He lines, is especially constraining for the hottest object in our sample J050632. In Table 4, we list for several models the expected equivalent width of the strongest He features when assuming a solar helium abundance. The solar He abundance is a lower limit for the actual abundance as an increase of the helium abundance in the envelope of our stars of up to $20 \%$ can be expected during the AGB evolution (e.g., Cristallo et al. 2009, 2011).

An excitation analyses of $\mathrm{Fe} \mathrm{I}$ is a traditional temperature sensor. Towards lower metallicities, higher effective temperatures, and lower gravities, the increasing UV radiation field and decreased rate of electron collisions may create an overionisation and over-excitation of iron with respect to the LTE description (Rentzsch-Holm 1996; Mashonkina et al. 2011). The same effect can be found in the abundances of other neutral elements, especially those of the iron peak. Observationally, these non-LTE effects have been noted in, amongst others, the classical Cepheid $\delta$ Cep (Kovtyukh \& Andrievsky 1999), and some 
Table 4. Expected equivalent widths of the strongest $\mathrm{He}$ lines (at $4471.50,5875.65$ and $5876.50 \AA$ ), and iron abundances for the best atmosphere model for a range of effective temperatures for J050632.

\begin{tabular}{|c|c|c|c|c|c|}
\hline \multirow{2}{*}{$\begin{array}{l}T_{\text {eff }} \\
(\mathrm{K})\end{array}$} & \multicolumn{3}{|c|}{ Expected EW (mÅ) } & \multirow{2}{*}{$\begin{array}{c}\log \epsilon_{\mathrm{FeI}}^{a} \\
\quad(\mathrm{dex})\end{array}$} & \multirow{2}{*}{$\begin{array}{c}\log \epsilon_{\mathrm{FeII}}{ }^{a} \\
(\mathrm{dex})\end{array}$} \\
\hline & 4471.50 & 5875.65 & 5876.50 & & \\
\hline 6250 & 0.7 & 0.3 & 0.3 & $5.69 \pm 0.23$ & $6.20 \pm 0.23$ \\
\hline 6500 & 4.6 & 1.1 & 1.2 & $5.89 \pm 0.20$ & $6.25 \pm 0.20$ \\
\hline 6750 & 10.5 & 2.8 & 3.0 & $6.09 \pm 0.18$ & $6.28 \pm 0.15$ \\
\hline 7000 & 17.6 & 5.6 & 6.0 & $6.34 \pm 0.17$ & $6.37 \pm 0.12$ \\
\hline
\end{tabular}

Notes. ${ }^{(a)}$ The listed errors are the line-to-line scatter. The Fe I abundance is based on the measurements of 63 lines and that of Fe II on 30.

post-AGB stars (Takeda et al. 2007; Gorlova et al. 2012). This means that the Teff we estimate from the excitation analyses of Fe I may be too high. At these temperatures this photoionisation scarcely alters the abundance of Fe II, with differences of less than 0.01 dex (Mashonkina et al. 2011) in the relevant temperature regime of our programme stars. The effective temperature deduced from a general excitation analysis of the Fe II lines is, however, less accurate because of the smaller number of available features and the lower spread in excitation potentials in optical Fe II lines.

Hydrogen line profiles do not show a strong dependence on metallicity of the stellar photosphere and we obtained the effective temperature of our stars by comparing their Balmer profiles with the synthetic spectra of Coelho et al. (2005). When estimating the appropriate effective temperature, we focused on the shape of the wings, as the depth of the feature may deviate from the synthetic spectra. This is the case because the core of the line is formed in the surface layers of the photosphere where the standard atmosphere models are less accurate due to the occurrence of surface phenomena and the boundary conditions of the models. We assume that the temperature and density gradient of our objects are similar to a model in hydrostatic equilibrium, despite the fact that the stars are pulsating.

For the given effective temperature, we first determined $\log g$ by imposing ionisation equilibrium for iron and, when available, other elements with lines of diferent ions. Only lines with an equivalent width of less than $150 \mathrm{~m} \AA$ were used, as the equivalent width of stronger lines is no longer strongly correlated with the abundance of the element, because they lay on the flat part of the curve of growth. We tried to estimate the Fe I abundance by extrapolating it to an equivalent width of $0 \mathrm{~m} \AA$ as described in Kovtyukh \& Andrievsky (1999), but the relatively large scatter on abundance and equivalent width of our lines rendered this impossible. The average abundance was used instead. We changed the metallicity of the model according to the obtained Fe II abundance. Finally, we estimated the microturbulent velocity $\xi_{t}$ in steps of $0.5 \mathrm{~km} \mathrm{~s}^{-1}$ by demanding that the abundance derived for the individual Fe II features does not depend on their reduced equivalent width. This procedure was repeated until the results converged towards a single model.

The final atmosphere models for our sample can be found in Table 5 and their corresponding synthetic Balmer profiles in Fig. 2. The final atmosphere models for both spectra of J052043 show a slight discrepancy in preferred gravities. Because of the coupling of this parameter with the effective temperature, this indicates a small temperature difference as well. As models with ionisation equilibrium between Fe I and Fe II require higher values of $\log g$ at higher effective temperatures, this confirms that J052043 became slightly cooler in December than it was in

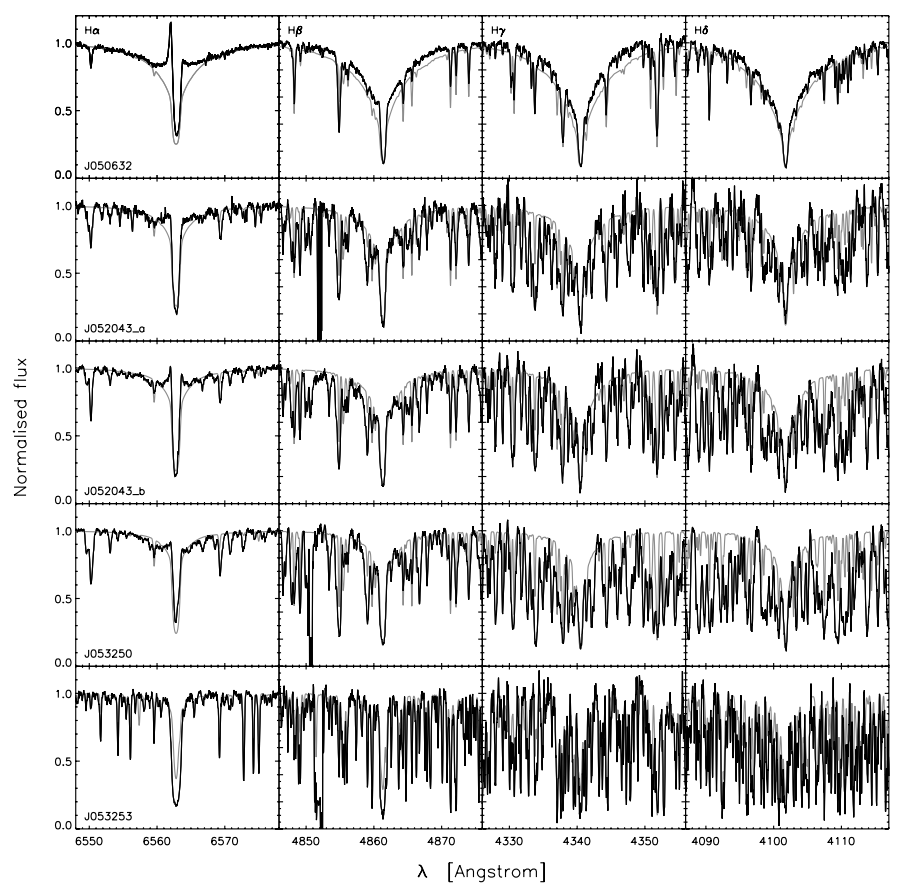

Fig. 2. Comparison with the synthetic spectra of Coelho et al. (2005). We show for each spectrum the first four Balmer lines and the synthetic spectrum of the final atmosphere model (see also Table 5). All synthetic spectra have been convolved with a Gaussian to match the resolution of the observed.

Table 5. Final atmosphere models.

\begin{tabular}{lcccc}
\hline \hline Object & $\begin{array}{c}T_{\text {eff }} \\
(\mathrm{K})\end{array}$ & $\begin{array}{c}\log g \\
(\mathrm{dex})\end{array}$ & $\begin{array}{c}\xi_{t} \\
\left(\mathrm{~km} \mathrm{~s}^{-1}\right)\end{array}$ & $\begin{array}{c}{[\mathrm{Fe} / \mathrm{H}]} \\
(\mathrm{dex})\end{array}$ \\
\hline J050632 & 6750 & 0.5 & 3.0 & -1.0 \\
J052043_a & 5750 & 0.5 & 3.0 & -1.0 \\
J052043_b & 5750 & 0.0 & 3.0 & -1.0 \\
J053250 & 5500 & 0.0 & 3.0 & -1.0 \\
J053253 & 4750 & 2.5 & 2.5 & -0.5 \\
\hline
\end{tabular}

November. The errors on the different atmosphere parameters are estimated to be the grid steps and therefore no interpolation of models and/or new models were computed.

\subsection{Spectrum synthesis}

The abundances of some elements could not be determined on the basis of isolated lines. An estimate of these abundances was nevertheless obtained through spectrum synthesis. To match the synthetic line profiles with the observed ones, we need a good description of the line-broadening which is the combined effect of instrumental, macroturbulent, and rotational broadening. Because the latter is thought to be small in F-G supergiants, and its effect on the spectra is difficult to distinguish from the macroturbulent broadening, we neglect it in our calculations. The macroturbulent broadening as the only unknown broadening parameter in the line profile fitting procedure.

\subsubsection{The macroturbulent broadening $\xi_{\mathrm{m}}$}

Based on the results Reyniers et al. (2004) obtained for the C-rich, Galactic post-AGB star IRAS 06530-0213, we expect that a single value for the macroturbulent broadening will not be sufficient to fit all lines in a spectrum equally well. This is 


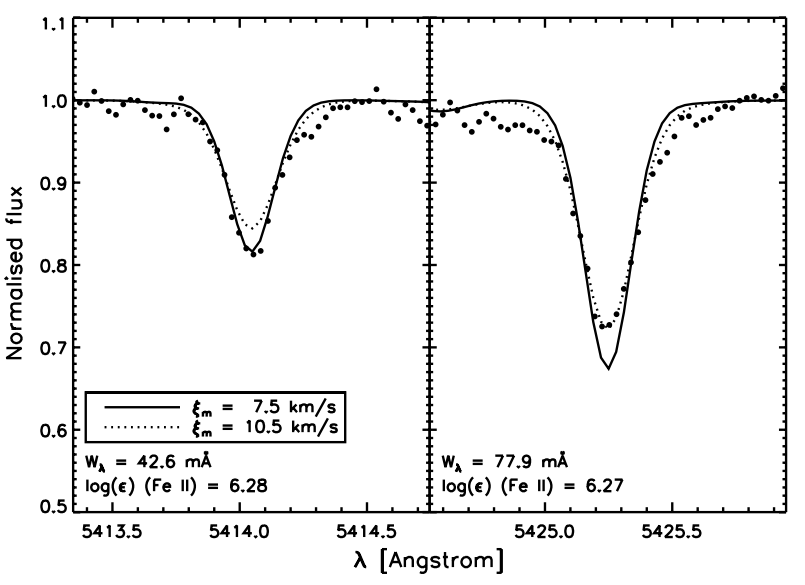

Fig. 3. Illustration of the necessity of different values of the macroturbulence at different reduced equivalent widths. We display two Fe II lines from the spectrum of J050632 from the same spectral region and with a similar abundance derived from their equivalent width. Their macroturbulent broadening factor differs, however, by $3 \mathrm{~km} \mathrm{~s}^{-1}$.

Table 6. Fitting parameters of the relation between the macroturbulent broadening and the reduced equivalent width.

\begin{tabular}{lccccc}
\hline \hline Object & Slope & Intersection & $\sigma^{a}$ & Corr. Coeff. & $N_{\text {lines }}$ \\
\hline J050632 & 2.2 & 20.6 & 2.2 & 0.60 & 107 \\
J052043_a & 5.4 & 30.4 & 2.3 & 0.79 & 21 \\
J052043_b & 3.5 & 25.1 & 1.9 & 0.78 & 52 \\
J053250 & 3.3 & 22.8 & 1.7 & 0.64 & 30 \\
J053253 & 1.0 & 8.9 & 2.2 & 0.26 & 58 \\
\hline
\end{tabular}

Notes. ${ }^{(a)}$ Standard deviation with respect to the fitted trend.

illustrated in Fig. 3 were we show two Fe II lines in the spectrum of J050632 with similar abundances, as derived from their equivalent width, and from the same spectral region. The two lines clearly require a different macroturbulent broadening factor. The reason for this dependence is not clear, but it is suggested that this is an optical depth effect where the stronger lines are on average being formed at lower geometrical depth (Reyniers et al. 2004). As similar trends have been found in HD 172481 (Reyniers \& Van Winckel 2001) and all other objects in our sample, this behaviour is certainly not exceptional. Most authors probably do not notice this effect as usually only a few lines or lines in the proximity of the feature at hand are investigated to determine the macroturbulent broadening.

We determined the macroturbulent broadening by a $\chi^{2}$ minimisation of the difference between a radial-tangential broadening profile (Gray 2008) that is corrected for the instrumental and microscopic broadening, and the observed feature. As the equivalent width remains unchanged while the line profile is broadened, we used the derived mean abundance of each element and left the macroturbulent broadening as the only free parameter to fit the line profile. We varied the broadening velocity in steps of $0.5 \mathrm{~km} \mathrm{~s}^{-1}$. Only the best, unblended lines of different elements and ionisation states were used in this procedure.

The resulting relation between the macroturbulent broadening and the reduced equivalent width for J050632 is shown as an example in Fig. 4. More information on the fitting parameters of this relation in the other spectra is listed in Table 6. The clear difference for ionised and neutral species that is observed in Reyniers et al. (2004), is not recovered for our spectra and the slopes we find are much smaller than their slope of 10.9 .

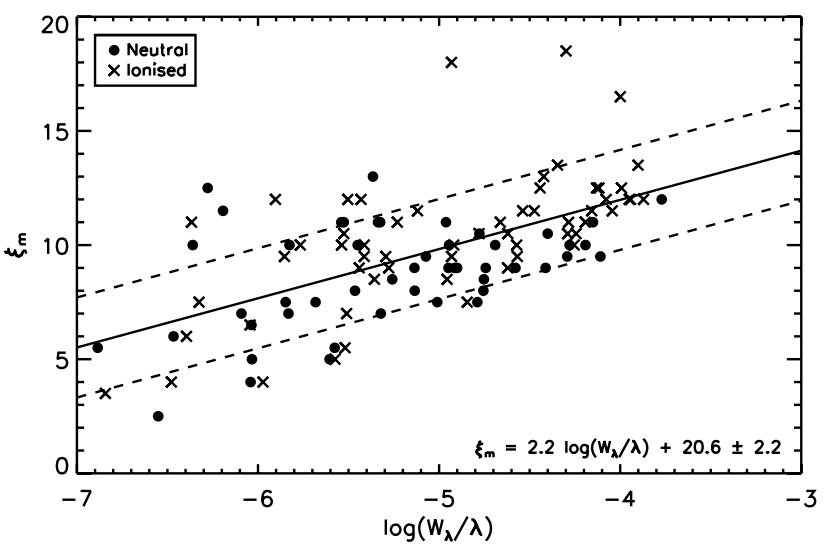

Fig. 4. Macroturbulent broadening $\xi_{m}$ of J050632 as a function of reduced equivalent width $\log \left(W_{\lambda} / \lambda\right)$. The dashed lines are the standard deviation on the mean trend.

\subsubsection{Blending}

All the single lines used to obtain specific abundances were checked individually to avoid the possibility that unrecognised blends could cause an overestimate of the abundance. For all used and presumably single lines, a list of all potential neighbouring lines was obtained from the VALD (Kupka et al. 1999) database. Their expected equivalent widths were computed based on the mean abundances found for every chemical element.

For each used blend, a list of all neighbouring lines in an interval of $1 \AA$ was extracted from VALD. We calculated the appropriate macroturbulent broadening with the relations from Sect. 4.2.1 for the total equivalent width of the line, as the element under consideration is the dominant contributor of the blend. This induces a possible error, as this relation is calculated for unblended lines, but it is unclear how it should be adapted in the case of blended lines. The synth driver of MOOG and all known abundances were used to perform the spectrum synthesis. The final abundance was obtained by a $\chi^{2}$ minimisation of the difference between the observed spectrum and the model in the spectral range used to calculate the equivalent width of the line. The abundances of $\mathrm{S}, \mathrm{Na}, \mathrm{Zn}, \mathrm{Ba}$, and $\mathrm{Sm}$ were based on blended lines.

\subsection{Elements beyond the Ba-peak}

Reyniers \& Van Winckel (2003) detected gadolinium, ytterbium, lutetium, and maybe also tungsten in the spectra of three Galactic, s-process enriched post-AGB stars. Inspired by their findings, we performed a specific systematic search for lines of elements beyond the Ba-peak. We focused on the elements from gadolinium ( $\mathrm{Gd}, Z=64)$ up to lead $(\mathrm{Pb}, Z=82)$, as the important features of elements with lower atomic numbers were already included in our original list (see Sect. 4.1).

For each element, the atomic data of all features in the optical spectrum were acquired from VALD (Kupka et al. 1999). The strongest expected lines were determined by imposing an ad hoc high abundance and calculating the corresponding equivalent widths for the appropriate atmosphere model. We then ordered the lines according to expected relative strength and systematically checked the obtained spectra for the presence of the spectral lines.

We discarding all lines for which (1) the nearest line in the spectrum was not centred on the given wavelength 
within $1 \mathrm{~km} \mathrm{~s}^{-1}$; (2) the signal to noise in the spectral region was too low to recognize a genuine spectral line; or (3) the line was located in a crowded region where the error on the position of the continuum appeared too large. For the remaining features, a list of lines of elements with already known abundance in the interval, was extracted from VALD and the equivalent width of these features determined with MOOG. Only lines of which about ninety percent of the observed equivalent width could not be explained by the elements of which the abundance was already known at that stage, were kept in the sample. After this analyses, we compared the abundances derived from all lines of the specific element. Features with too high an equivalent width were discarded as blends with unrecognised contributors. A final quality check was performed by recalculating the expected equivalent width of the strongest lines of the element at hand with this new final abundance, and comparing this to what is seen in the spectra. As the atomic data of all lines listed in VALD are not equally reliable, we only executed the latter test for elements of which the abundance is based on at most three lines.

The strongest features of all heavy s-process elements are concentrated in the low $\mathrm{S} / \mathrm{N}$ region from 3280 to $4519 \AA$ and we were plagued by the low $\mathrm{S} / \mathrm{N}$ of our spectra in the blue. Nonetheless, we were able to determine the abundances for a range of heavy s-process elements. Lines of $\mathrm{Gd}(64), \mathrm{Tb}(65)$, Dy(66), Er(68), Tm(69), Yb(70), Hf(72), Ta(73), W(74), Hg(80) were positively itentified in many of the objects, and the results are given in the abundance Tables 7-10.

\subsection{Hyperfine structure}

We investigated the influence of hyperfine splitting ( $\mathrm{hfs}=$ the splitting of spectral lines caused by nucleon-electron spin interaction) on the derived abundances of all odd- $Z$ elements from $\mathrm{La}(Z=57)$ to $\mathrm{Lu}(Z=71)$. Because hfs causes extra desaturation, it will only affect the abundance determination when this is based on strong lines that are situated on the flat part of the curve of growth.

To make sure our results are independent of the method used for abundance determination, we first recalculated the abundances of the elements by use of spectrum synthesis (see Sect. 4.2.2), treating each feature as a single line. This process was then repeated taking hfs into account, with the final goal of comparing the acquired abundances. For each spectral line, we obtained the energy levels corresponding to its wavelength from the National Institute of Standards and Technology ${ }^{2}$ (NIST). Appropriate values of the hfs constants $A$ and $B$ were obtained from Lawler et al. (2001a) for La II $(Z=57)$, from Sneden et al. (2009) for Pr II $(Z=59)$, from Lawler et al. (2001b) for Eu II $(Z=63)$, from Lawler et al. (2001c) for Tb II $(Z=65)$, and from Sneden et al. (2003) for Lu II $(Z=71)$. We ignored $\operatorname{Pm}(Z=61$, no stable isotope), Ho $(Z=67$, no detections $)$ and $\operatorname{Tm}(Z=69$, no $A, B$ constants found) in our calculations. Because the electric quadrupole interaction ( $B$ constant) has a much smaller effect on the line component pattern than the magnetic dipole interaction ( $A$ constant), it is often neglected and we set its value to zero if it could not be found in the literature. The relative strengths of the different hfs components were calculated with the equations given in Condon \& Shortley (1935).

A real, but small effect of at most $\Delta_{\mathrm{ab}}=\log \epsilon_{\mathrm{hfs}}-\log \epsilon_{\mathrm{no} \mathrm{hfs}}=$ -0.10 dex is noticed in some features, but falls within the original error margin on the abundances, and is dwarfed by other uncertainties like e.g., the exact value $\log g f$, the placement of

\footnotetext{
2 http://www.nist.gov/pml/index.cfm
}

Table 7. Abundance results for J050632.10-714229.8.

\begin{tabular}{|c|c|c|c|c|c|c|c|}
\hline \multirow[b]{3}{*}{ Ion } & \multicolumn{6}{|c|}{ J050632 } & \multirow{3}{*}{$\begin{array}{l}\text { Sun } \\
\log \epsilon\end{array}$} \\
\hline & \multicolumn{3}{|c|}{$\begin{array}{l}T_{\text {eff }}=6750 \mathrm{~K} \\
\log g=0.5\end{array}$} & \multicolumn{3}{|c|}{$\begin{array}{l}\xi_{t}=3.0 \mathrm{~km} \mathrm{~s}^{-1} \\
{[\mathrm{Fe} / \mathrm{H}]=-1.22}\end{array}$} & \\
\hline & $N$ & $\begin{array}{c}{\overline{W_{\lambda}}}^{a} \\
(\mathrm{~mA})\end{array}$ & $\log \epsilon$ & $\sigma_{\mathrm{ltl}}$ & {$[\mathrm{el} / \mathrm{Fe}]$} & $\sigma_{\text {tot }}$ & \\
\hline $\mathrm{CI}$ & 21 & 47 & 8.37 & 0.08 & 1.16 & 0.11 & 8.43 \\
\hline O I & 3 & $s s$ & 8.18 & & 0.91 & 0.24 & 8.69 \\
\hline Mg I & 1 & 84 & 6.38 & & -0.03 & 0.32 & 7.60 \\
\hline $\mathrm{Si}$ I & 1 & 5 & 6.88 & & 0.59 & 0.32 & 7.51 \\
\hline S I & 1 & $s s$ & 5.93 & & 0.03 & 0.27 & 7.12 \\
\hline $\mathrm{Ca} \mathrm{I}$ & 11 & 23 & 4.98 & 0.10 & -0.14 & 0.32 & 6.34 \\
\hline Sc II & 8 & 58 & 1.72 & 0.09 & -0.20 & 0.1 & 3.15 \\
\hline Ti II & 18 & 74 & 3.82 & 0.11 & 0.09 & 0.08 & 4.95 \\
\hline V II & 1 & 74 & 2.48 & & -0.23 & 0.22 & 3.93 \\
\hline CrI & 1 & 89 & 3.97 & & -0.45 & 0.40 & 5.64 \\
\hline Cr II & 17 & 40 & 4.46 & 0.11 & 0.05 & 0.04 & 5.64 \\
\hline $\mathrm{Fe} I$ & 63 & 36 & 6.09 & 0.18 & -0.18 & 0.29 & 7.50 \\
\hline Fe II & 30 & 44 & 6.28 & 0.15 & 0.00 & 0.04 & 7.50 \\
\hline $\mathrm{Ni}$ I & 3 & 8 & 4.62 & 0.03 & -0.38 & 0.30 & 6.22 \\
\hline $\mathrm{Zn}$ I & 1 & 12 & 3.26 & & -0.08 & 0.35 & 4.56 \\
\hline $\mathrm{Y} \mathrm{II}_{\mathrm{I}}$ & 8 & 88 & 2.53 & 0.05 & 1.54 & 0.11 & 2.21 \\
\hline Zr II & 12 & 41 & 2.66 & 0.10 & 1.30 & 0.09 & 2.58 \\
\hline Ba II & 1 & SS & 2.24 & & 1.28 & 0.34 & 2.18 \\
\hline La II & 17 & 32 & 1.36 & 0.08 & 1.48 & 0.23 & 1.10 \\
\hline Ce II & 28 & 48 & 1.68 & 0.10 & 1.33 & 0.21 & 1.58 \\
\hline Pr II & 8 & 15 & 0.91 & 0.09 & 1.42 & 0.30 & 0.72 \\
\hline $\mathrm{Nd}$ II & 20 & 36 & 1.38 & 0.08 & 1.18 & 0.30 & 1.42 \\
\hline Sm II & 1 & ss & 0.05 & & 0.29 & 0.33 & 0.96 \\
\hline Eu II & 2 & 30 & -0.19 & 0.17 & 0.51 & 0.27 & 0.52 \\
\hline Gd II & 19 & 22 & 0.81 & 0.11 & 0.96 & 0.16 & 1.07 \\
\hline Dy II & 5 & 23 & 0.73 & 0.07 & 0.88 & 0.21 & 1.10 \\
\hline $\mathrm{Yb} \mathrm{II}^{b}$ & 2 & 5 & 1.31 & 0.12 & 1.70 & 0.15 & 0.84 \\
\hline Ta II ${ }^{b}$ & 1 & 9 & 0.71 & & 2.05 & 0.23 & -0.12 \\
\hline
\end{tabular}

Notes. For each element, we list the number of lines involved in the abundance determination, the average equivalent width of these lines, the deduced abundance, the standard deviation on this abundance, and the $[\mathrm{el} / \mathrm{Fe}]$ value with its corresponding error (see text for details). The solar abundances in the last column are from Asplund et al. (2009). If no solar photospheric abundance value was available, as was the case for $\mathrm{Ta}, \mathrm{Re}$, and $\mathrm{Hg}$, we took the meteoric equivalent. ${ }^{(a)}$ The indication ss denotes that the abundance was deduced with the use of spectrum synthesis (see Sect. 4.2.2). ${ }^{(b)}$ These abundances should be treated with caution as they are based on small features when compared to the noise level, and are upper limits only.

the continuum or undetected blends. We conclude that the effect of hfs on the derived abundance is very small.

\section{Abundance results}

In Tables 7-10 we present the complete abundance analysis results of the stars in our sample, the details on all lines used in this analysis can be found in five catalogues that are available at the CDS and contain the following information. For each catalogue, Col. 1 gives the name of the ion a certain line belongs to, Col. 2 contains its rest frame wavelength, Col. 3 lists its lower excitation potential. The logarithm of the oscillator strength can be found in Col. 4, Col. 5 gives the measured equivalent width, and the abundance deduced from this line is mentioned in Col. 6 .

We determined the errors on the $[\mathrm{el} / \mathrm{Fe}]$ abundances in Tables 7-10 with the method described in Deroo et al. (2005), and the alternative procedure for estimating the error induced by the parameter uncertainty of the atmosphere model from 
E. van Aarle et al.: Detailed abundance study of four s-process enriched LMC post-AGB stars

Table 8. Same as Table 7, but for the spectra of J052043.86-692341.0 taken in November and December 2008.

\begin{tabular}{|c|c|c|c|c|c|c|c|c|c|c|c|c|c|}
\hline \multirow{3}{*}{ Ion } & \multicolumn{6}{|c|}{ J052043_a } & \multirow{3}{*}{$\begin{array}{c}\text { Sun } \\
\log \epsilon\end{array}$} & \multicolumn{6}{|c|}{ J052043_b } \\
\hline & \multicolumn{3}{|c|}{$\begin{array}{l}T_{\text {eff }}=5750 \mathrm{~K} \\
\log g=0.5\end{array}$} & \multicolumn{3}{|c|}{$\begin{array}{c}\xi_{t}=3.0 \mathrm{~km} \mathrm{~s}^{-1} \\
{[\mathrm{Fe} / \mathrm{H}]=-1.24}\end{array}$} & & \multicolumn{3}{|c|}{$\begin{array}{l}T_{\text {eff }}=5750 \mathrm{~K} \\
\log g=0.0\end{array}$} & & \multicolumn{2}{|c|}{$\begin{array}{l}\xi_{t}=3.0 \mathrm{~km} \mathrm{~s}^{-1} \\
{[\mathrm{Fe} / \mathrm{H}]=-1.15}\end{array}$} \\
\hline & $N$ & $\begin{array}{r}\overline{\overline{W \lambda}^{a}} \\
(\mathrm{m \AA})\end{array}$ & $\log \epsilon$ & $\sigma_{1 \mathrm{lt}}$ & {$[\mathrm{el} / \mathrm{Fe}]$} & $\sigma_{\text {tot }}$ & & $N$ & 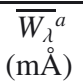 & $\log \epsilon$ & $\sigma_{\mathrm{ltl}}$ & {$[\mathrm{el} / \mathrm{Fe}]$} & $\sigma_{\text {tot }}$ \\
\hline$\overline{C I}$ & 13 & 71 & 8.72 & 0.07 & 1.53 & 0.20 & 8.43 & 11 & 49 & 8.59 & 0.14 & 1.30 & 0.18 \\
\hline O I & 1 & ss & 8.30 & & 0.85 & 0.35 & 8.69 & 1 & 15 & 8.40 & & 0.86 & 0.35 \\
\hline $\mathrm{NaI}$ & 1 & ss & 5.18 & & 0.18 & 0.35 & 6.24 & & & & & & \\
\hline $\operatorname{Mg} I$ & 1 & 26 & 6.29 & & -0.07 & 0.35 & 7.60 & 1 & 36 & 6.54 & & 0.09 & 0.32 \\
\hline $\mathrm{Si}$ I & 8 & 18 & 6.82 & 0.17 & 0.55 & 0.29 & 7.51 & 4 & 25 & 6.75 & 0.16 & 0.38 & 0.32 \\
\hline Si II & 2 & 55 & 6.89 & 0.05 & 0.61 & 0.31 & 7.51 & 2 & 16 & 6.86 & 0.09 & 0.50 & 0.31 \\
\hline S I & 2 & 26 & 6.32 & 0.11 & 0.43 & 0.20 & 7.12 & 2 & ss & 6.31 & 0.06 & 0.33 & 0.18 \\
\hline $\mathrm{Ca} I$ & 12 & 55 & 5.20 & 0.10 & 0.10 & 0.32 & 6.34 & 12 & 67 & 5.44 & 0.13 & 0.25 & 0.18 \\
\hline $\mathrm{Ca}$ II & & & & & & & 6.34 & 1 & 10 & 5.46 & & 0.27 & 0.28 \\
\hline Sc II & 3 & 80 & 1.96 & 0.13 & 0.04 & 0.16 & 3.15 & 4 & 79 & 1.94 & 0.05 & -0.06 & 0.16 \\
\hline Ti I & & & & & & & 4.95 & 1 & 4 & 3.61 & & -0.19 & 0.39 \\
\hline Ti II & & & & & & & 4.95 & 6 & 94 & 3.90 & 0.12 & 0.09 & 0.10 \\
\hline Cr I & & & & & & & 5.64 & 2 & 26 & 4.27 & 0.01 & -0.22 & 0.38 \\
\hline Cr II & 11 & 56 & 4.54 & 0.09 & 0.13 & 0.05 & 5.64 & 12 & 62 & 4.57 & 0.11 & 0.08 & 0.05 \\
\hline $\mathrm{Fe} \mathrm{I}$ & 63 & 59 & 6.16 & 0.19 & -0.11 & 0.34 & 7.50 & 73 & 60 & 6.25 & 0.16 & -0.10 & 0.29 \\
\hline $\mathrm{Fe}$ II & 18 & 66 & 6.26 & 0.11 & 0.00 & 0.04 & 7.50 & 23 & 68 & 6.35 & 0.17 & 0.00 & 0.05 \\
\hline Ni I & 9 & 22 & 4.86 & 0.13 & -0.12 & 0.33 & 6.22 & 13 & 26 & 4.96 & 0.12 & -0.11 & 0.27 \\
\hline Zn I & 1 & 37 & 3.08 & & -0.24 & 0.40 & 4.56 & & & & & & \\
\hline Y II & 2 & sS & 2.63 & & 1.86 & 0.21 & 2.21 & 2 & 129 & 2.78 & 0.15 & 1.71 & 0.21 \\
\hline Zr II & 5 & 46 & 2.81 & 0.16 & 1.47 & 0.12 & 2.58 & 4 & 70 & 2.88 & 0.15 & 1.44 & 0.16 \\
\hline La II & 13 & 94 & 1.97 & 0.09 & 2.10 & 0.20 & 1.10 & 7 & 89 & 1.80 & 0.03 & 1.85 & 0.21 \\
\hline Ce II & 5 & 66 & 2.03 & 0.13 & 1.68 & 0.16 & 1.58 & 5 & 63 & 2.11 & 0.06 & 1.68 & 0.16 \\
\hline Pr II & 7 & 79 & 1.62 & 0.12 & 2.14 & 0.21 & 0.72 & 11 & 59 & 1.39 & 0.14 & 1.82 & 0.23 \\
\hline Nd II & 13 & 85 & 2.14 & 0.10 & 1.96 & 0.21 & 1.42 & 20 & 95 & 2.19 & 0.12 & 1.92 & 0.24 \\
\hline Sm II & 3 & 127 & 1.38 & 0.10 & 1.66 & 0.24 & 0.96 & 2 & 137 & 1.29 & 0.11 & 1.47 & 0.25 \\
\hline Eu II & 2 & 74 & 0.52 & 0.09 & 1.24 & 0.21 & 0.52 & 1 & 52 & 0.36 & & 0.99 & 0.26 \\
\hline Gd II & 4 & 17 & 1.04 & 0.13 & 1.21 & 0.17 & 1.07 & 9 & 24 & 1.12 & 0.09 & 1.20 & 0.15 \\
\hline Tb II & 1 & 14 & 0.36 & & 1.30 & 0.29 & 0.30 & 1 & 25 & 0.53 & & 1.38 & 0.29 \\
\hline Dy II & 4 & 8 & 1.25 & 0.12 & 1.39 & 0.21 & 1.10 & 1 & 18 & 1.22 & & 1.27 & 0.30 \\
\hline Er II & & & & & & & 0.92 & & 13 & 1.27 & 0.08 & 1.50 & 0.16 \\
\hline Yb II & 3 & 12 & 1.95 & 0.14 & 2.35 & 0.12 & 0.84 & 5 & 15 & 1.80 & 0.13 & 2.11 & 0.07 \\
\hline Hf II & 1 & 13 & 1.94 & & 2.33 & 0.21 & 0.85 & 1 & 12 & 1.33 & & 1.63 & 0.24 \\
\hline
\end{tabular}

Reyniers et al. (2007). To estimate this error, we calculated the abundance difference for a certain element for our preferred model and for the most appropriate model to fit our data with a $250 \mathrm{~K}$ higher/lower temperature and 0.5 higher/lower gravity $(\log g)$. The total uncertainty on the $[\mathrm{el} / \mathrm{Fe}]$ abundances can be found in Tables 7-10. It is the quadratic sum of the uncertainties on the mean due to line-to-line scatter $\left(\sigma_{1 t 1}\right)$, the abundance ratio uncertainties induced by the model parameters $\left(\sigma_{\text {teff }}, \sigma_{\log g}\right)$, and the uncertainty on the $\mathrm{Fe}$ abundance $\left(\sigma_{\mathrm{Fe}}\right)$ :

$\sigma_{\text {tot }}=\sqrt{\left(\frac{\sigma_{\mathrm{ltl}}}{\sqrt{N_{\mathrm{el}}}}\right)^{2}+\left(\sigma_{\mathrm{teff}}\right)^{2}+\left(\sigma_{\log g}\right)^{2}+\left(\frac{\sigma_{\mathrm{Fe}}}{\sqrt{N_{\mathrm{Fe}}}}\right)^{2}}$.

If less than 5 lines were available, a line-to-line scatter of 0.2 dex was applied. All results are presented graphically in Figs. 5 and 6. We now highlight the most important abundance characteristics.

\subsection{Metallicity}

J050632, J052043, and J053250 are strongly metal deficient with $\mathrm{a}[\mathrm{Fe} / \mathrm{H}]$ of -1.2 (see Table 11 ). J053253 is only mildly metal deficient with $[\mathrm{Fe} / \mathrm{H}]=-0.5$. Overall, the metallicities of the stars in the $\mathrm{LMC}$ range from $[\mathrm{Fe} / \mathrm{H}]=-2.0$ up to $[\mathrm{Fe} / \mathrm{H}]=-0.3$, with most objects having $[\mathrm{Fe} / \mathrm{H}] \sim-0.5$ (Geisler et al. 2009). The fact that three of the four programme stars display metallicities on the low-end of the detected range, indicates that these are old, evolved objects with low initial masses.

For each object, the other iron peak elements follow this deficiency but the number of detected useable lines is much lower. The relative abundances of nickel in J050632 and of zinc in J052043 and J053253 are somewhat low.

The non-LTE ionisation effect in J050632, J052043, and J053250 can be seen in the abundances of the other elements of the iron peak if results of more than one ionisation state are present. Both titanium and chromium show this bias, with derived abundances on the basis of neutral lines being significantly lower than the abundances based on ionised lines. This nonLTE effect is not seen is silicon and calcium where abundances based on differen ions are in accordance. Only in the spectrum of J053250 does the silicon abundances deviate from this trend with Si I having an abundance of 6.85, while it is 7.12 for Si II. In the abundance pattern of J053253, the non-LTE effect is not witnessed, and the obtained abundance of $\mathrm{CrI}$ is even higher than that of $\mathrm{Cr}$ II.

\subsection{C/O-ratio}

All objects for which the determination of the carbon abundance was possible, are clearly enriched, with values of $[\mathrm{C} / \mathrm{Fe}]$ ranging 
Table 9. Same as Table 7, but for J053250.69-713925.8.

\begin{tabular}{|c|c|c|c|c|c|c|c|}
\hline \multirow{3}{*}{ Ion } & \multicolumn{6}{|c|}{ J053250 } & \multirow{3}{*}{$\begin{array}{c}\text { Sun } \\
\log \epsilon\end{array}$} \\
\hline & \multicolumn{3}{|c|}{$\begin{array}{l}T_{\text {eff }}=5500 \mathrm{~K} \\
\log g=0.0\end{array}$} & \multicolumn{3}{|c|}{$\begin{array}{l}\xi_{t}=3.0 \mathrm{~km} \mathrm{~s}^{-1} \\
{[\mathrm{Fe} / \mathrm{H}]=-1.22}\end{array}$} & \\
\hline & $N$ & $\begin{array}{c}\overline{{\overline{W_{\lambda}}}^{a}} \\
(\mathrm{~m} \AA)\end{array}$ & $\log \epsilon$ & $\sigma_{\mathrm{lt} 1}$ & {$[\mathrm{el} / \mathrm{Fe}]$} & $\sigma_{\text {tot }}$ & \\
\hline$\overline{C I}$ & 9 & 47 & 8.74 & 0.11 & 1.53 & 0.21 & 8.43 \\
\hline O I & 1 & ss & 8.35 & & 0.88 & 0.38 & 8.69 \\
\hline $\mathrm{NaI}$ & 1 & 23 & 5.01 & & -0.01 & 0.30 & 6.24 \\
\hline Si I & 4 & 35 & 6.84 & 0.12 & 0.55 & 0.25 & 7.51 \\
\hline Si II & 1 & 29 & 7.15 & & 0.86 & 0.39 & 7.51 \\
\hline S I & 1 & ss & 6.05 & & 0.15 & 0.22 & 7.12 \\
\hline Ca I & 11 & 74 & 5.21 & 0.13 & 0.09 & 0.28 & 6.34 \\
\hline Sc II & 4 & 88 & 1.92 & 0.05 & -0.01 & 0.16 & 3.15 \\
\hline Ti II & 7 & 123 & 3.93 & 0.20 & 0.20 & 0.12 & 4.95 \\
\hline V II & 1 & 134 & 2.77 & & 0.06 & 0.21 & 3.93 \\
\hline $\mathrm{CrI}$ & 2 & 26 & 3.96 & 0.16 & -0.46 & 0.40 & 5.64 \\
\hline Cr II & 12 & 52 & 4.50 & 0.12 & 0.08 & 0.05 & 5.64 \\
\hline Fe I & 58 & 58 & 6.12 & 0.21 & -0.16 & 0.31 & 7.50 \\
\hline Fe II & 17 & 70 & 6.28 & 0.10 & 0.00 & 0.03 & 7.50 \\
\hline Ni I & 9 & 28 & 4.81 & 0.13 & -0.19 & 0.30 & 6.22 \\
\hline Y II & 1 & 118 & 2.66 & & 1.67 & 0.27 & 2.21 \\
\hline Zr II & 2 & 67 & 2.74 & 0.03 & 1.35 & 0.17 & 2.58 \\
\hline La II & 7 & 106 & 1.91 & 0.13 & 2.03 & 0.23 & 1.10 \\
\hline Ce II & 8 & 88 & 2.26 & 0.15 & 1.91 & 0.17 & 1.58 \\
\hline PrII & 13 & 63 & 1.37 & 0.14 & 1.87 & 0.22 & 0.72 \\
\hline $\mathrm{Nd}$ II & 9 & 91 & 2.22 & 0.13 & 2.03 & 0.22 & 1.42 \\
\hline Eu II & 1 & 68 & 0.41 & & 1.11 & 0.26 & 0.52 \\
\hline Gd II & 7 & 11 & 1.18 & 0.13 & 1.33 & 0.15 & 1.07 \\
\hline Dy II & 4 & 26 & 1.39 & 0.10 & 1.52 & 0.19 & 1.10 \\
\hline Er II & 2 & 12 & 1.49 & 0.09 & 1.79 & 0.17 & 0.92 \\
\hline $\operatorname{Tm}_{\text {II }}^{b}$ & 1 & 4 & 0.87 & & 1.99 & 0.20 & 0.10 \\
\hline Yb II & 3 & 10 & 1.68 & 0.05 & 2.06 & 0.12 & 0.84 \\
\hline $\mathrm{Hf} \mathrm{II}^{b}$ & 2 & 7 & 1.25 & 0.08 & 1.62 & 0.18 & 0.85 \\
\hline
\end{tabular}

from 1.5 in J050632 to 2.5 in J053250. Surprisingly, large enhancement in oxygen is also observed in these objects with [O/Fe] around 0.8 in J052043, J050632 and J05325. The C/O number ratios remain relatively moderate as a consequence of this large oxygen enhancement, with values of 2.6 to 1.5 (see Table 11). No conclusions on both the carbon and oxygen abundance of J053253 are possible as at the low effective temperature of this object, no lines of these elements are visible in the optical wavelength domain.

\section{3. $\alpha$-elements}

The mean of the $[\mathrm{el} / \mathrm{Fe}]$ ratios for the $\alpha$-elements $\mathrm{Mg}, \mathrm{Si}$, $\mathrm{S}, \mathrm{Ca}$, and $\mathrm{Ti}$, falls between 0.1 and 0.3 for all objects (see Table 11). This is slightly deficient when compared to stars of the same metallicities in the Galaxy, but consistent with the $\alpha$-enhancement expected for LMC stars at their respective metallicities (Pompéia et al. 2008). Because the $\alpha$-elements are predicted to be mainly produced in high-mass type II supernovae, Pompéia et al. (2008) interpret the moderate $\alpha$-enhancement at low metallicites in the LMC as indicative of a stronger influence of type Ia supernovae. This also accords with the more extended star formation history in the LMC than in the Galaxy.

\section{4. s-process elements}

In general, the s-process elements observed in evolved stars are subdivided into three groups, depending on the number of neutrons in their nuclei. The light s-process elements have neutron
Table 10. Same as Table 7, but for J053253.51-695915.1.

\begin{tabular}{|c|c|c|c|c|c|c|c|}
\hline \multirow[b]{3}{*}{ Ion } & \multicolumn{6}{|c|}{ J053253 } & \multirow{3}{*}{$\begin{array}{c}\text { Sun } \\
\log \epsilon\end{array}$} \\
\hline & \multicolumn{3}{|c|}{$\begin{array}{l}T_{\text {eff }}=4750 \mathrm{~K} \\
\log g=2.5\end{array}$} & \multicolumn{3}{|c|}{$\begin{array}{l}\xi_{t}=2.5 \mathrm{~km} \mathrm{~s}^{-1} \\
{[\mathrm{Fe} / \mathrm{H}]=-0.54}\end{array}$} & \\
\hline & $N$ & $\begin{array}{c}{\overline{W_{\lambda}}}^{a} \\
(\mathrm{~m} \AA)\end{array}$ & $\log \epsilon$ & $\sigma_{\text {ltl }}$ & {$[\mathrm{el} / \mathrm{Fe}]$} & $\sigma_{\text {tot }}$ & \\
\hline $\mathrm{Na} \mathrm{I}$ & 2 & 74 & 5.92 & 0.02 & 0.22 & 0.18 & 6.24 \\
\hline Mg I & 1 & 139 & 7.13 & & 0.07 & 0.21 & 7.60 \\
\hline Al I & 2 & 56 & 6.03 & 0.08 & 0.13 & 0.17 & 6.45 \\
\hline Si I & 15 & 53 & 7.24 & 0.10 & 0.31 & 0.29 & 7.51 \\
\hline $\mathrm{Ca} \mathrm{I}$ & 6 & 134 & 5.94 & 0.10 & 0.15 & 0.20 & 6.34 \\
\hline $\mathrm{Sc}$ II & 9 & 82 & 2.85 & 0.09 & 0.24 & 0.35 & 3.15 \\
\hline Ti II & 8 & 113 & 4.60 & 0.09 & 0.19 & 0.38 & 4.95 \\
\hline Cr I & 1 & 70 & 5.58 & & 0.48 & 0.24 & 5.64 \\
\hline Cr II & 8 & 57 & 5.43 & 0.13 & 0.33 & 0.51 & 5.64 \\
\hline Mn I & 4 & 87 & 5.23 & 0.18 & 0.34 & 0.15 & 5.43 \\
\hline $\mathrm{Fe} \mathrm{I}$ & 60 & 98 & 7.03 & 0.13 & 0.08 & 0.02 & 7.50 \\
\hline $\mathrm{Fe}$ II & 18 & 70 & 6.96 & 0.08 & 0.00 & 0.00 & 7.50 \\
\hline $\mathrm{Ni} \mathrm{I}$ & 25 & 88 & 5.75 & 0.12 & 0.07 & 0.09 & 6.22 \\
\hline $\mathrm{ZnI}$ & 1 & $s S$ & 3.76 & & -0.26 & 0.44 & 4.56 \\
\hline Y II & 8 & 74 & 2.03 & 0.14 & 0.36 & 0.34 & 2.21 \\
\hline Zr II & 6 & 59 & 2.76 & 0.15 & 0.73 & 0.33 & 2.58 \\
\hline Mo I & 1 & 39 & 2.10 & & 0.76 & 0.37 & 1.88 \\
\hline La II & 12 & 53 & 1.54 & 0.12 & 0.98 & 0.29 & 1.10 \\
\hline Ce II & 10 & 71 & 1.74 & 0.13 & 0.70 & 0.31 & 1.58 \\
\hline Pr II & 7 & 37 & 1.37 & 0.12 & 1.19 & 0.32 & 0.72 \\
\hline $\mathrm{Nd}$ II & 22 & 58 & 1.80 & 0.10 & 0.92 & 0.30 & 1.42 \\
\hline Sm II & 1 & 100 & 1.34 & 0.18 & 0.92 & 0.35 & 0.96 \\
\hline Eu II & 2 & 48 & 0.86 & 0.06 & 0.88 & 0.39 & 0.52 \\
\hline Gd II & 4 & 39 & 1.33 & 0.15 & 0.81 & 0.33 & 1.07 \\
\hline Tb II & 2 & 21 & 0.98 & 0.06 & 1.22 & 0.35 & 0.30 \\
\hline W I & 1 & 47 & 1.48 & 0.20 & 1.17 & 0.36 & 0.85 \\
\hline
\end{tabular}

Notes. For this cooler star, Fe I is the dominant ionisation stage.

numbers around the magic number $50(\mathrm{Y}, \mathrm{Zr}, \ldots)$, and the neutron numbers of the heavy s-process elements are distributed around the magic number $82(\mathrm{Ba}, \mathrm{La}, \mathrm{Ce}, \mathrm{Pr}, \mathrm{Nd}, \mathrm{Sm}, \ldots)$. The third and final group corresponds to the double magic state of neutron number 126 and consists of lead only $\left({ }^{208} \mathrm{~Pb}\right.$ contains 126 neutrons and 82 protons), as it is seen as the end product of the s-process nucleosynthesis.

From Table 11 and Figs. 5 and 6 it is clear that all objects are strongly enriched in s-process elements, which confirms the post third dredge-up status of our stars. The enrichment in s-process elements is the lowest in J053253, where $[\mathrm{s} / \mathrm{Fe}]=0.85$. J053250 and J052043 are s-process enhanced at almost the level of IRAS 06530-0213 (Reyniers et al. 2004), and IRAS 05341+0852 (Van Winckel \& Reyniers 2000), which are, with respectively $[\mathrm{s} / \mathrm{Fe}]=2.1$ and $[\mathrm{s} / \mathrm{Fe}]=2.2$, some of the most s-process enriched intrinsic Galactic objects known to date.

\section{Luminosities and initial masses}

With the best atmosphere models as followed from the abundance analysis, we are now able to make a better estimate of the luminosities, and consequently initial masses of the stars in our sample.

\subsection{Luminosity}

In Fig. 7 we show the SEDs of the different stars, where the appropriate atmosphere model (see Sect. 4.1) was fitted to the 
E. van Aarle et al.: Detailed abundance study of four s-process enriched LMC post-AGB stars

Table 11. The $\mathrm{C} / \mathrm{O}$ number ratio, metallicity, enhancement in $\alpha$-elements and the s-process indexes for the stars in our sample.

\begin{tabular}{lccccc}
\hline \hline Object & $\mathrm{C} / \mathrm{O}$ & {$[\mathrm{Fe} / \mathrm{H}]$} & {$[\alpha / \mathrm{Fe}]$} & {$[\mathrm{s} / \mathrm{Fe}]$} & {$[\mathrm{s} / \mathrm{Fe}]_{\mathrm{La}, \mathrm{Nd}}$} \\
\hline J050632 & $1.5 \pm 0.3$ & $-1.22 \pm 0.16$ & $0.11 \pm 0.09$ & $1.17 \pm 0.09$ & $1.38 \pm 0.07$ \\
J052043_a & $2.6 \pm 1.3$ & $-1.24 \pm 0.12$ & $0.26 \pm 0.08$ & $1.87 \pm 0.06$ & $1.84 \pm 0.07$ \\
J052043_b & $1.6 \pm 0.9$ & $-1.15 \pm 0.17$ & $0.23 \pm 0.07$ & $1.75 \pm 0.06$ & $1.74 \pm 0.07$ \\
J053250 & $2.5 \pm 0.7$ & $-1.22 \pm 0.11$ & $0.26 \pm 0.07$ & $1.83 \pm 0.07$ & $1.78 \pm 0.07$ \\
J053253 & & $-0.54 \pm 0.09$ & $0.18 \pm 0.07$ & $0.85 \pm 0.07$ & $0.75 \pm 0.08$ \\
\hline Object & {$[\mathrm{ls} / \mathrm{Fe}]$} & {$[\mathrm{hs} / \mathrm{Fe}]$} & {$[\mathrm{hs} / \mathrm{Fe}]_{\mathrm{La}, \mathrm{Nd}}$} & {$[\mathrm{hs} / \mathrm{ls}]$} & {$[\mathrm{hs} / \mathrm{ls}]_{\mathrm{La}, \mathrm{Nd}}$} \\
\hline J050632 & $1.42 \pm 0.05$ & $1.06 \pm 0.14$ & $1.33 \pm 0.14$ & $-0.36 \pm 0.15$ & $-0.09 \pm 0.15$ \\
J052043_a & $1.65 \pm 0.10$ & $1.95 \pm 0.07$ & $1.84 \pm 0.07$ & $0.30 \pm 0.13$ & $0.39 \pm 0.13$ \\
J052043_b & $1.58 \pm 0.10$ & $1.79 \pm 0.08$ & $1.91 \pm 0.09$ & $0.21 \pm 0.13$ & $0.34 \pm 0.14$ \\
J053250 & $1.53 \pm 0.11$ & $1.96 \pm 0.08$ & $2.03 \pm 0.09$ & $0.43 \pm 0.14$ & $0.50 \pm 0.14$ \\
J053253 & $0.55 \pm 0.10$ & $0.94 \pm 0.10$ & $0.95 \pm 0.13$ & $0.39 \pm 0.14$ & $0.41 \pm 0.16$ \\
\hline
\end{tabular}
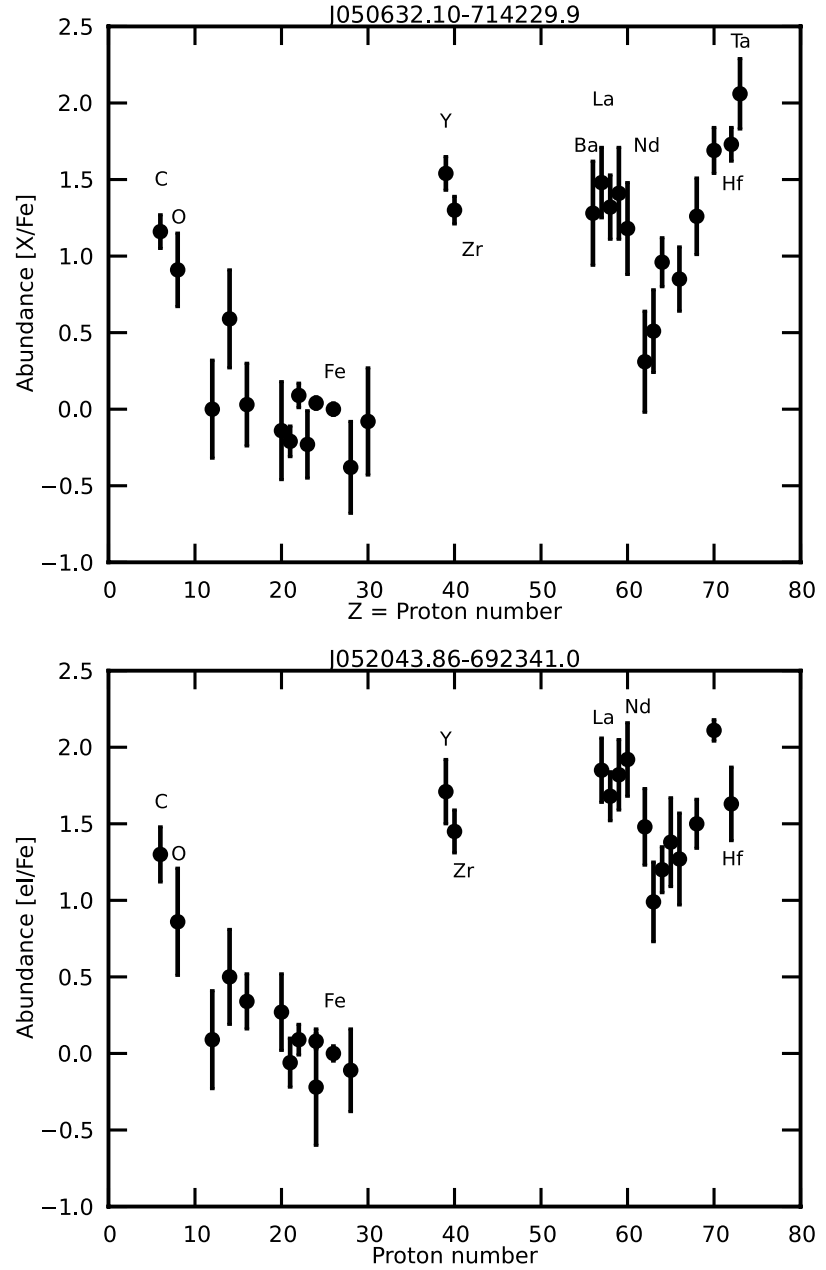

Fig. 5. Derived abundance patterns for J050622 (upper panel) and J052043, obtained with highest S/N spectrum (lower panel).

dereddened photometry. The $E(B-V)$ was determined by minimising the difference between the scaled model and the dereddened photometric data, excluding the infrared where thermal emission of dust contributes to the total fluxmeasurement. Errors in the luminosity and the reddening are dominated by the uncertainty in the parameters of the model atmosphere which are more important than the uncertainties of the photometric data points themselves. We therefore ran our SED fitting routine for all input models within the uncertainties of the spectral analyses.

In Table 12 we compare our luminisity estimates of the different program stars, with those obtained by integrating the raw
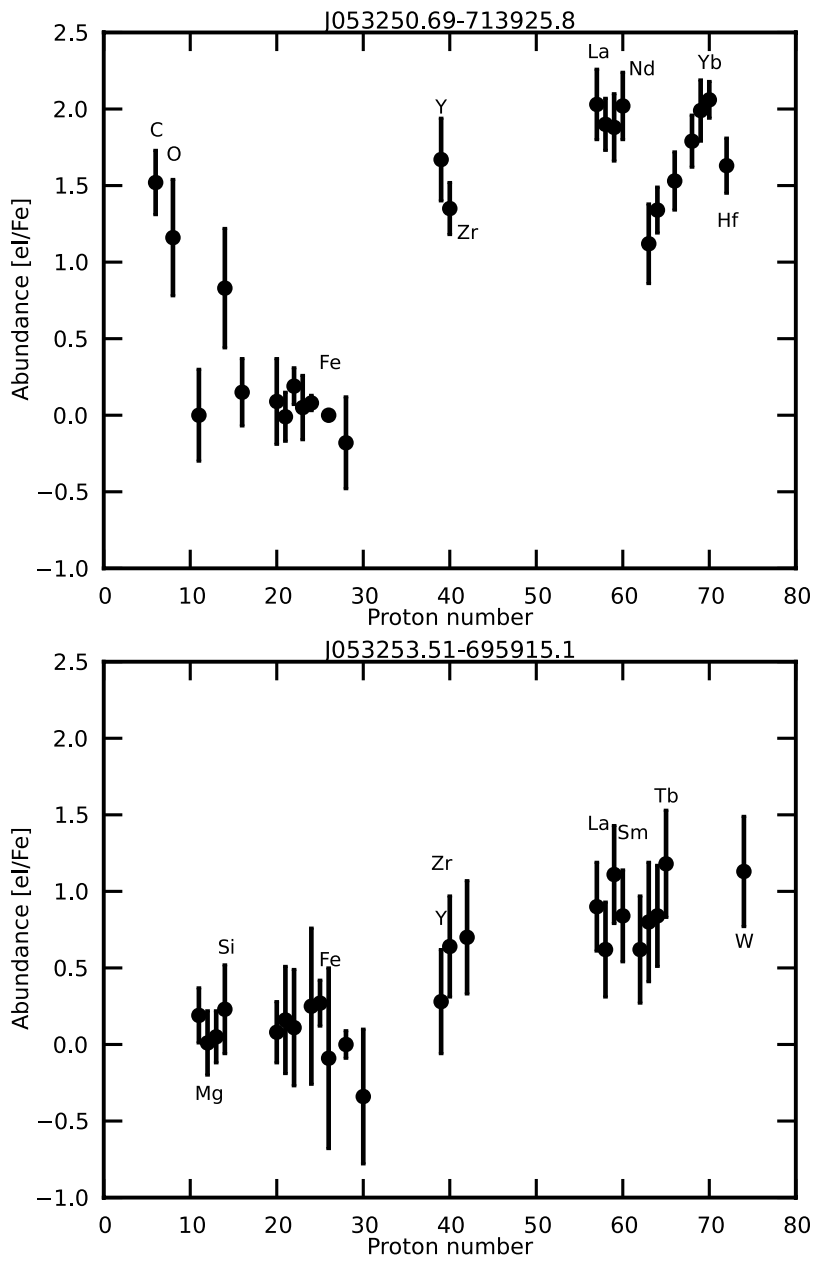

Fig. 6. Derived abundance patterns for J053250 (upper panel) and J053253, obtained with highest S/N spectrum (lower panel).

photometric data over the full SED (in the table given in the column raw SED integral). The luminosity based on integral of the raw data holds a good estimate of the luminosity based on the model atmosphere fit. This indicates that the circumstellar shell is spherically symmetric, and that the reddening caused by the interstellar medium is negligible. With these assumptions, the interstellar reddening can be neglected, while the extinction caused by the circumstellar shell is accounted for by the luminosity of the infrared excess.

In van Aarle et al. (2011), we used the spectral type to determine a range of possible effective temperatures and concluded 

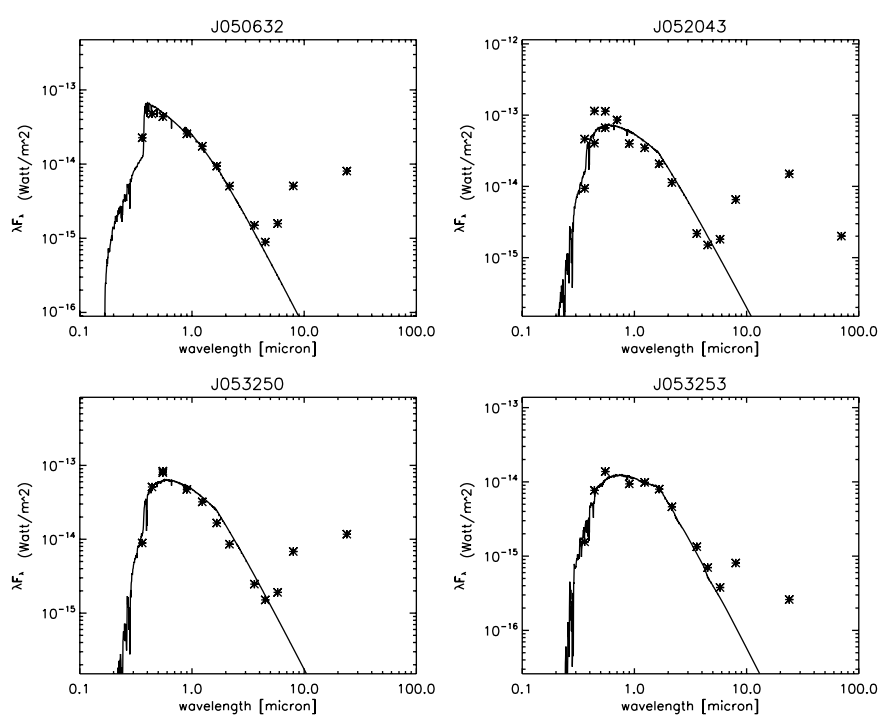

Fig. 7. Final SEDs of the stars in our program. We fitted the dereddened photometry to the rescaled atmosphere models, the parameters of which are based on the abundance analysis.

Table 12. Comparison of luminosity and reddening values obtained with our different computation methods.

\begin{tabular}{lcccc}
\hline \hline Object & \multicolumn{3}{c}{ Final results } & SED integral. \\
& $T_{\text {eff }}$ & $L$ & $E(B-V)$ & $\begin{array}{c}L \\
\left(L_{\odot}\right)\end{array}$ \\
& $(\mathrm{K})$ & $\left(L_{\odot}\right)$ & & 5570 \\
$\mathrm{~J} 050632$ & $6750 \pm 250$ & $5400 \pm 700$ & $0.05 \pm 0.06$ & 5730 \\
$\mathrm{~J} 052043$ & $5750 \pm 250$ & $8700 \pm 1000$ & $0.43 \pm 0.07$ & 4700 \\
$\mathrm{~J} 053250$ & $5500 \pm 250$ & $6500 \pm 1000$ & $0.50 \pm 0.08$ & 1150 \\
$\mathrm{~J} 053253$ & $4750 \pm 250$ & $1400 \pm 300$ & $0.21 \pm 0.12$ & \\
\hline
\end{tabular}

simultaneously on the best reddening factor and effective temperature by fitting atmosphere models to the dereddened photometry. We used, however, only models with metallicity -0.3 , which is too high for three of the four stars we discuss here in detail. These objects are intrinsically bluer than the used models, which leads to an overestimation of the effective temperature, and consequently a too high reddening and luminosity. This lead in van Aarle et al. (2011) to an overestimate of the luminosity and the integral over the raw photometry is a better estimate of the true luminosity. In Kamath et al. (2013) we report on our low-resolution spectral fitting routine to obtain more accurate model photospheres on the basis of low-resolution spectra.

\subsection{Initial mass}

The more accurate values of effective temperature and luminosity allow us to estimate the initial mass of the stars in our sample. In Fig. 8 the position of the objects in the HR diagram is shown, together with the evolutionary tracks from Vassiliadis $\&$ Wood (1994). The tracks in the upper plot have a metallicity of $Z=0.001$ which is the lowest value in the model grid of Vassiliadis \& Wood (1994) but is still somewhat higher than what we find for J050632 ( $Z=0.0007)$, J052043 ( $Z=0.0008)$, and $\mathrm{J} 053250(Z=0.0007)$. The tracks in the lower plot correspond to $Z=0.004$, which is the grid value that approaches the metallicity of $\mathrm{J} 053253(Z=0.0040)$ the best. We converted the metallicities of the stars in our program to $Z$-values by computing this parameter with its definition, under the assumptions that the Fe-abundance remained the same and that all initial
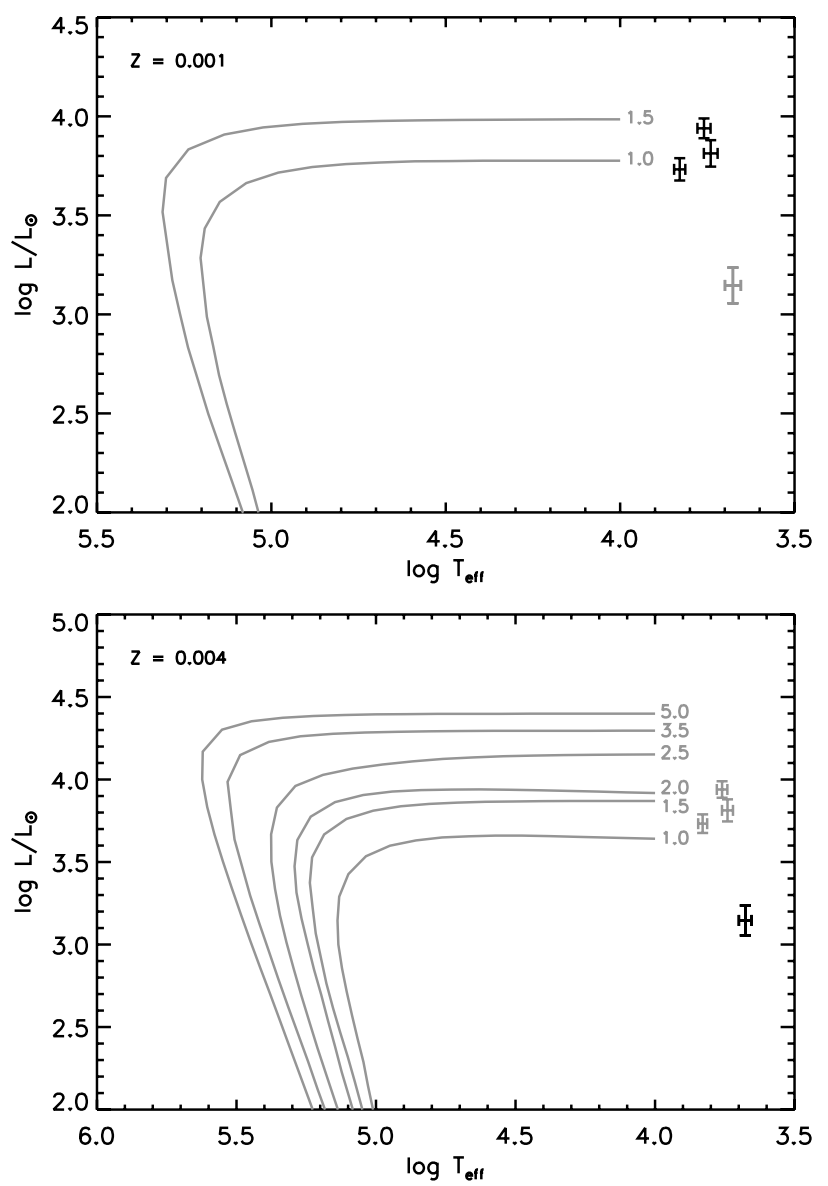

Fig. 8. Position of our stars in the HR diagram. The displayed evolutionary tracks are from Vassiliadis \& Wood (1994), with metallicities of $Z=0.001$ (upper panel), and $Z=0.004$ (lower panel), and are labelled with their initial mass in $M_{\odot}$. The location of each star is shown in black in the appropriate diagram and in grey in the other to compare the position of the different data points.

abundances, except for those of hydrogen and helium, scale with the iron abundance.

From Fig. 8 it follows that the initial masses of the stars in our sample are all smaller than about $1.5 M_{\odot}$, under the assumption that these objects follow the single star evolutionary tracks. J052043 appears to be the most massive, followed by J053250 and J050632, although the difference is small. J053253 is located below the evolutionary tracks, which would indicate an significantly lower initial mass. This is difficult to understand as objects with masses below $0.8 M_{\odot}$ have main sequence life times that are larger that the Hubble time. We conclude that J053253 is likely not evolving along the single-star evolutionary tracks depicted here. The evolutionary status of this object is discussed further in Sect. 8.

\section{Neutron exposure}

Four observational indexes are traditionally defined to describe the s-process overabundances as well as the s-process distribution: [s/Fe], [ls/Fe], [hs/Fe], and [hs/ls]. The specific elements taken into account when calculating these indexes, vary from author to author, and mainly depend on which abundances could be determined and the reliability of these results. To be consistent with earlier publications (e.g., Reyniers et al. 2004), we follow the suggestion from Busso et al. (1995) and define the ls-index 
as the mean of the relative abundances of $\mathrm{Y}$, and $\mathrm{Zr}$, and the hsindex as the mean of $\mathrm{Ba}, \mathrm{La}, \mathrm{Nd}$, and $\mathrm{Sm}$. Consequently, [s/Fe] is the mean of the six elements and $[\mathrm{hs} / \mathrm{ls}]=[\mathrm{hs} / \mathrm{Fe}]-[\mathrm{ls} / \mathrm{Fe}]$.

The s-process indexes of our sample stars are listed in Table 11. Missing abundances of specific s-process elements need to be determined, however. For this we scaled the abundance distribution prediction of the most appropriate AGB model (see Sect. 9) to the nearest element in atomic mass with a measured abundance. We then assume that the object displays the same abundance ratio for elements nearest in atomic mass. The Ba abundance of J052043_a, J052043_b, J053250, and J053253, and the Sm abundance of J053250 had to be estimated this way. The four heavy s-process elements all originate from the same iron seeds and we assume that their relative abundances only depend on their cross-sections for neutron capture. Leaving the missing abundances out is not a reliable alternative as due to the odd-even effect, the overabundances of these four elements are not the same. Alternative indexes of comparable quality but based on the abundances of $\mathrm{La}$, and $\mathrm{Nd}$ only (Reyniers et al. 2004) can also be obtained, and we list these for comparison.

Observationally, it is well known that there is a strong correlation between the $[\mathrm{s} / \mathrm{Fe}]$ and $[\mathrm{hs} / \mathrm{ls}]$ indexes (e.g., Van Winckel \& Reyniers 2000; Reyniers et al. 2004), with objects with a higher $[\mathrm{s} / \mathrm{Fe}]$ index showing also a higher [hs/ls] ratio. The $[\mathrm{s} / \mathrm{Fe}]$ index forms an indication of the third dredge-up efficiency, although this parameter is also influenced by the mass loss history of the star and the envelope mass at dredge-ups. The [hs/ls] index represents the effectiveness of the neutron irradiation, which is related to the ratio of the number of neutrons available to the iron seed nuclei. Considering that the stars in our sample are of similar metallicity, this parameter would be determined by the abundance difference between ${ }^{13} \mathrm{C}$, the main neutron seed, and ${ }^{14} \mathrm{~N}$, the main neutron poison in the stellar interior (e.g. Herwig 2005). This difference, however, depends on many unconstrained parameters. The neutron production in AGB models is fuelled by the injection of protons into the intershell, which creates the ${ }^{13} \mathrm{C}$ pocket through ${ }^{12} \mathrm{C}(\mathrm{p}, \gamma)^{13} \mathrm{~N}\left(\beta^{+}\right)^{13} \mathrm{C}$, and allows the neutron producing reaction ${ }^{13} \mathrm{C}(\alpha, \mathrm{n}){ }^{16} \mathrm{O}$ to take place. The correlation therefore indicates that with increasing dredge-up efficiency, also the dredge-in of protons will increase as they are the primary cause of a higher neutron irradiation.

The correlation between the strength of the neutron irradiation, and the efficiency of the third dredge-up is graphically depicted in Fig. 9. Three of the four stars in our sample further confirm this assertion, but in J053253 the relatively high neutron irradiation only led to a mild enhancement in s-process elements. A simple, linear least-squares fit to all data points gives $[\mathrm{hs} / \mathrm{ls}]=0.55 \times[\mathrm{s} / \mathrm{Fe}]-0.78$, and a correlation coefficient of 0.52 . If we ignore $\mathrm{J} 053253$, this relation becomes $[\mathrm{hs} / \mathrm{ls}]=0.92 \times[\mathrm{s} / \mathrm{Fe}]-1.43$, with a correlation coefficient of 0.92 . This is comparable to the correlation coefficient of 0.96 that corresponds to the original data (Reyniers et al. 2004).

The $[\mathrm{hs} / \mathrm{ls}]$ index is expected to increase with decreasing metallicity as a consequence of the larger number of neutrons per iron seed nucleus. This assumes, however, that the diffusion of protons into the $\mathrm{C}$-rich intershell itself is independent of the metallicity or parameters linked to this metallicity. Only a weak correlation with a broad intrinsic spread is observed between the neutron irradiation and the metallicity (e.g., Van Winckel \& Reyniers 2000; Reyniers et al. 2004), which indicates that other stellar parameters also determine the internal nucleosynthesis during the AGB evolution, and we are dealing with a large spread in ${ }^{13} \mathrm{C}$ pocket efficiencies in highly similar objects.

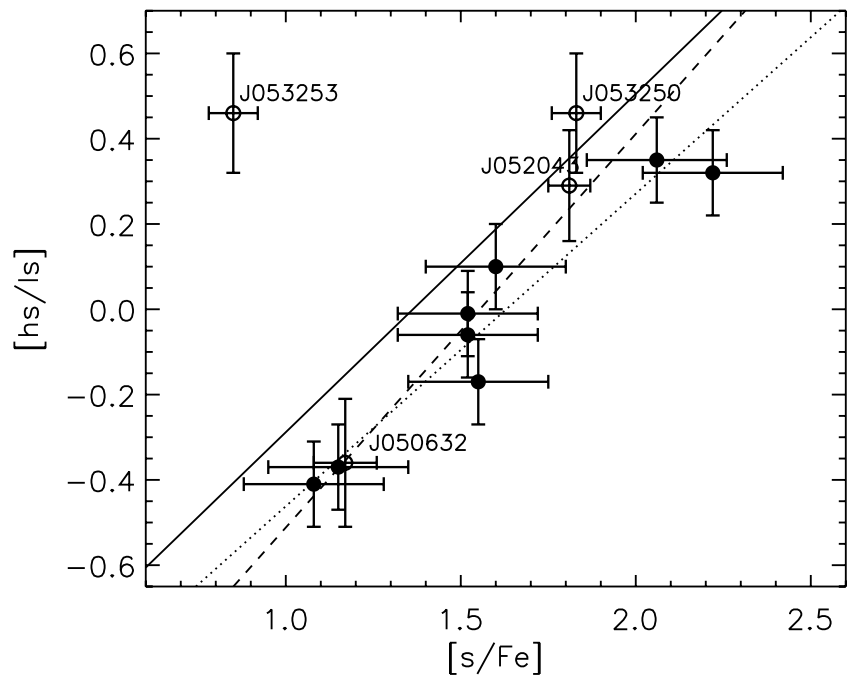

Fig. 9. Correlation between the total enrichment in s-process elements and the $[\mathrm{hs} / \mathrm{ls}]$ index. The objects of this paper are named. The others are the $21 \mu \mathrm{m}$ sources from Van Winckel \& Reyniers (2000), and the two enriched objects from Reyniers et al. (2004). The full line gives the least-squares fit to all data points, the dashed line to all data points except for J053253, and the dotted line, which is given for comparison, to all older data points.

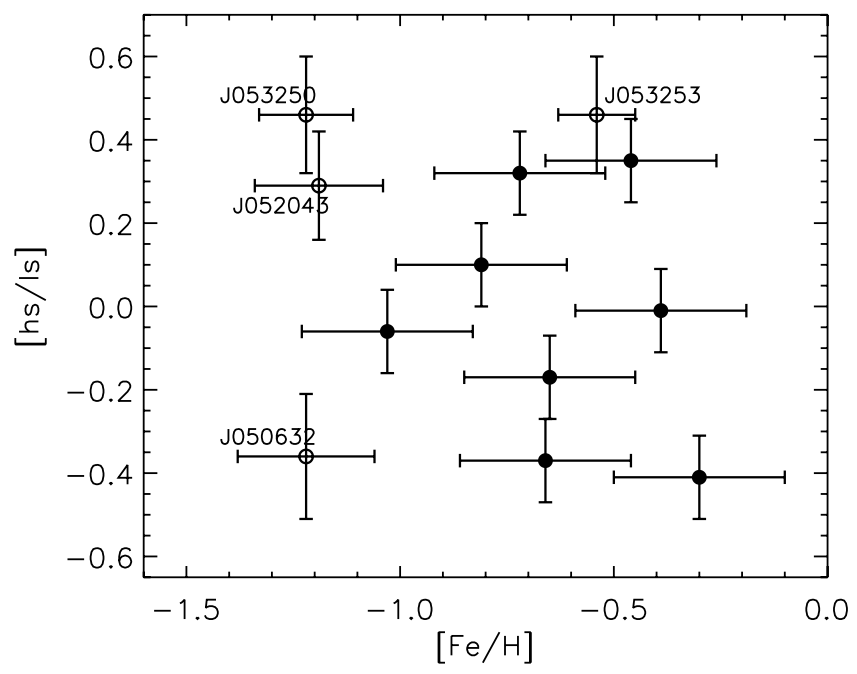

Fig. 10. [hs/ls] index as a function of the metallicity. The symbols are the same as in Fig. 9. No correlation is found between the metallicity and the $[\mathrm{hs} / \mathrm{ls}]$ index in this range of metallicities.

With their range of metallicities from -0.54 to -1.22 , our objects offer the possibility to extend this relation to lower values of $[\mathrm{Fe} / \mathrm{H}]$. In Fig. 10 we added the current sample of post-AGB stars to the objects from Van Winckel \& Reyniers (2000) and Reyniers et al. (2004) in a plot of [hs/ls] versus [Fe/H]. Our results confirm that there is no correlation between metallicity and the $[\mathrm{hs} / \mathrm{ls}]$ index in this range of metallicities.

\section{Is J053253 an extrinsic object?}

Objects that are enhanced in s-process elements, can have reached this status in two different ways. In the case of intrinsic post-AGB stars, the surface composition became s-process enriched by dredge-ups of nucleosynthetic products created in the stellar interior. In extrinsic objects, the enrichment is an effect of mass transfer of s-process elements from a further evolved 
companion to the star at hand. The former AGB star is now typically a cool white dwarf. Therefore, it is possible that the enhanced object itself is not yet a post-AGB star.

The strange position of J053253 in Figs. 8 and 9 suggests that the s-process enrichment in this object may be extrinsic: J053253 is much less luminous than can be expected from post-AGB evolutionary tracks, and it does not follow the strong correlation that is found between third dredge-up efficiency and neutron irradiation. There are, however, two arguments that contradict this interpretation. First of all, Tomkin et al. (1989) detected only two barium dwarfs in their survey of about 200 F-G dwarfs, which is comparable to the about one percent of RGB stars that is extrinsic (MacConnell et al. 1972). A similar number is hence expected for post-AGB stars, and it would be very surprising to encounter one in our sample of only four stars.

The second argument considers the dust that is seen in the system of J053253. This object clearly has a dust excess, although it is rather small when compared to the other stars described here. These excesses are generally not seen in extrinsic objects, as the circumstellar dust dissipates into the interstellar medium (ISM) on a timescale that is short compared to the evolutionary timescales. The relative proximity of the dust in the system of J053253, as follows from its colour, indicates that, if the material was lost in a wind, it must have been shed very recently. In this scenario we may conclude that J053253 is either indeed an intrinsic post-AGB star, be it at very low luminosity or it is a post-RGB star which happens to have had a dusty massloss event. A post-RGB status conflicts with internal s-process enhancement.

Another possible explanation for the deviating position of J053253 in Figs. 8 and 9 may be found in the shape of its SED (see Fig. 7). Although the dust in the system is cooler than is typically expected for a post-AGB object with a circumstellar disc, it radiates the bulk of its energy at wavelengths shorter than $24 \mu \mathrm{m}$. It is hence a valid possibility that the circumstellar material surrounding this object resides in a disc rather than in a freely expanding, detached shell. It is known from the Galactic sample of post-AGB stars with a circumstellar disc, that the AGB evolution has been cut short by a phase of strong binary interaction (Van Winckel et al. 2009; Gielen et al. 2011). Proof of this theory is found in the orbits that are detected, which are too small to accommodate an AGB star (Van Winckel et al. 2009; Gorlova et al. 2012), and the chemical composition of the dust, which consists of strongly processed silicates (Gielen et al. 2011). This truncation of the stellar evolution should translate itself in a subluminous object and should also alter the expected chemical composition of the photosphere: the $[\mathrm{s} / \mathrm{Fe}]$ index is supposed to be lower, because fewer third dredge-ups can take place, while the [hs/ls] index will remain similar as each third dredge-up brings heavy and light s-process elements to the surface.

\section{Comparison with AGB nucleosynthetic models}

The current theoretical models of the internal nucleosynthesis and the subsequent photospheric enrichment processes of AGB stars are sophisticated and rely on the complex interplay between mixing, nucleosynthesis, and mass loss (Busso et al. 1999; Herwig 2005). In these models, extensive nuclear networks have been included (e.g. Cristallo et al. 2009, 2011; Karakas 2010; Church et al. 2009; Goriely et al. 2008; Lugaro et al. 2012), non-convective mixing like mixing due to differential rotation and thermohaline mixing have been implemented (e.g. Siess et al. 2003; Siess 2007; Stancliffe \& Jeffery 2007; Angelou et al. 2011), the effect of deep mixing or extra mixing processes has been critically evaluated (e.g. Karakas 2010; Busso et al. 2010), and overshoot regimes have been explored in more detail to explain the appearance of the ${ }^{13} \mathrm{C}$ pocket as the main neutron source (e.g. Herwig 2005). The models are not self-consistent and overshoot regimes must be incorporated. The proton ingestion process is complex and detailed simulations at low metallicities in 3D yield clearly different results as the traditional 1D approaches (Stancliffe et al. 2011).

Tailored model computations for the individual stars as well as detailed comparison of the the different codes like we have done in De Smedt et al. (2012), fall outside the scope of this paper. The main discrepancy between the model abundances and the derived ones was that the predicted $\mathrm{C} / \mathrm{O}$ ratio was much too high compared to the observed one. Moreover, for that one star in the SMC (J004441.04-732136.4), the models fit the s-process distribution well, despite the fact that the absolute abundances were not well matched. The main conclusion of De Smedt et al. (2012) was that we found only a weak dependency of the theoretical predictions on the adopted stellar evolution code. With the newly analysed objects we want to investigate whether these discrepancies between AGB nucleosynthesis predictions and the observed chemical patters are a systematic feature and hence found in more objects.

For this general comparison, we used the nucleosynthetic AGB models from the online-database FRUITY ${ }^{3}$ (Franec Repository of Upgraded Isotopic Tables \& Yields, Cristallo et al. 2011). The models that are currently in the database focus on low mass AGB stars with initial masses between 1.5 and $3.0 M_{\odot}$ and metallicities $Z$ in the range from 0.001 to 0.02 .

We used the initial mass and metallicity estimates from Sect. 6.2 to determine the model that approximated these values the best for each object. All models have an initial mass of $1.5 M_{\odot}$ as this is the lowest value in the grid. As we suspect that J053253 is extrinsically enriched, we assume that the donor has this mass as well (see Sect. 6.2). We use a metallicity of $Z=0.001$ for J050632, J052043, and J053250 as also this value is on the lower edge of the grid, and $Z=0.003$ and 0.006 for J053253, because the metallicity of this object falls between these two values.

In Fig. 11 we show some characteristics of the models as a function of the number of third dredge-up episodes the star has undergone. The model with metallicity $Z=0.001$ reaches the post-AGB phase after fourteen third dredge-ups, while this already happens after respectively ten and eight for the models with $Z=0.003$ and 0.006 . We plotted the $\mathrm{C} / \mathrm{O}$ and ${ }^{12} \mathrm{C} /{ }^{13} \mathrm{C}$ ratio, as well as the $[\mathrm{s} / \mathrm{Fe}]$ and $[\mathrm{hs} / \mathrm{ls}]$ indexes as a function of the number of dredge-ups the star has undergone at that point.

Also for these stars, we confirm that the relative abundances of both $\mathrm{C}$ and $\mathrm{O}$ are badly predicted by the models: $\mathrm{C}$ is in general overpredicted while the model values for $\mathrm{O}$ remain well below the actual determined value. This is a fundamental problem of the models, as especially for the C-abundances, the model values lie well outside the error bars on our relative abundances. The combination of both deviations leads to a too high predicted $\mathrm{C} / \mathrm{O}$ ratio of the AGB models. From the first panel in Fig. 11, it follows that the model with $Z=0.001$ already estimates the corresponding observed $\mathrm{C} / \mathrm{O}$ ratios after the occurrence of only one third dredge-up. The $\mathrm{C} / \mathrm{O}$ ratio of the final model with this metallicity overrates the observed one by a factor $\sim 20$. As in De Smedt et al. (2012), the high observed O-abundance, which also contributes to the $\mathrm{C} / \mathrm{O}$ ratio, is not accounted for.

http://www.oa-teramo.inaf.it/fruity 

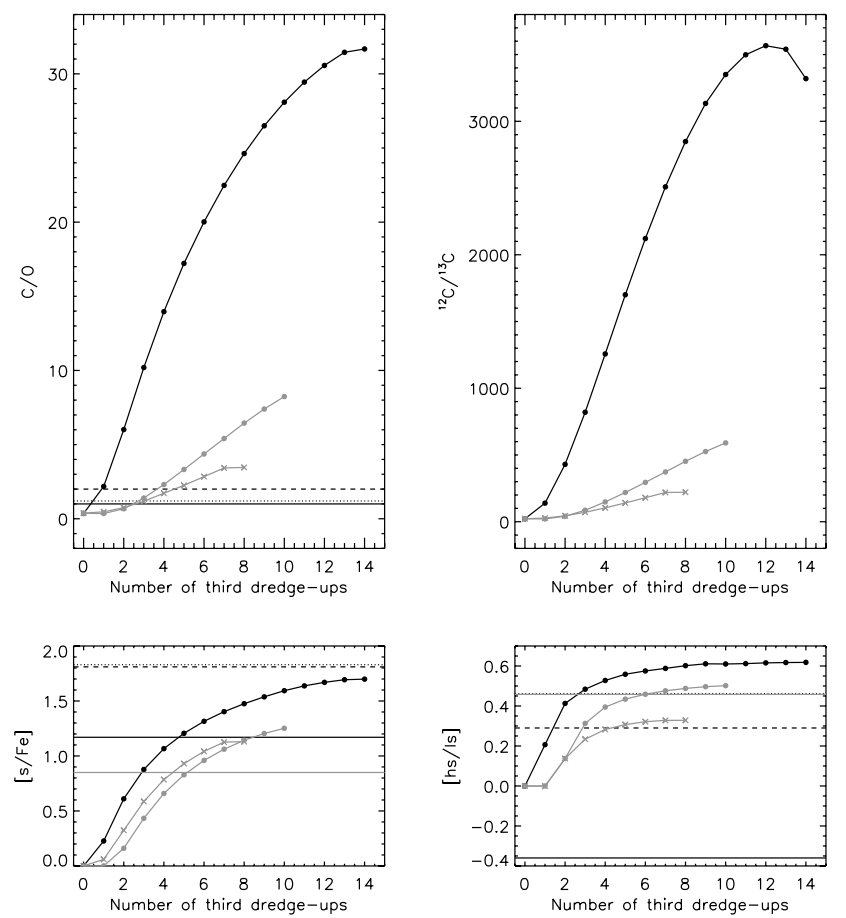

Fig. 11. Some characteristics of the used nucleosynthetic AGB models. We show the $\mathrm{C} / \mathrm{O}$ and ${ }^{12} \mathrm{C} /{ }^{13} \mathrm{C}$ ratio, and the $[\mathrm{s} / \mathrm{Fe}]$ and $[\mathrm{hs} / \mathrm{ls}]$ indexes as a function of the number of third dredge-ups the model has undergone. Each third dredge-up episode is represented by a dot or a cross, depending on the nucleosynthetic model, and they are linked for clarity. The model with metallicity $Z=0.001$ is shown as connected black dots, while the models with metallicity $Z=0.003$ and $Z=0.006$ are represented by grey dots and crosses respectively. The values obtained for the stars in our sample are overplotted as horizontal lines in the same colour (black,grey) as their most appropriate nucleosynthetic model. J050632 is represented by a full, black line, J052043 by a dashed, black line, J053250 by a dotted, black line, and J053253 by a full, grey line.

Our spectra do not allow deduction of the ${ }^{12} \mathrm{C} /{ }^{13} \mathrm{C}$ isotopic fractions, because the $\mathrm{CN}$ violet and red systems around 3875 and $6191 \AA$ are not detected at the $\mathrm{S} / \mathrm{N}$ of our spectra and the temperature of our stars. Our spectral range does not cover the additional $\mathrm{CN}$ red system at $6945 \AA$, and the wavelength region from 1-5 $\mu \mathrm{m}$, where several $\mathrm{CO}$ forests could be found. Determining the ${ }^{12} \mathrm{C} /{ }^{13} \mathrm{C}$ isotopic ratio is an observational challenge in these objects but it would allow a very good test on the predictive power of the AGB models.

For the objects with the highest s-process enhancement, J052043 and J053250, the comparison of the s-process distribution with theoretical models matches relatively well, but the nucleosynthetic models underestimate the actual abundances of the light s-process elements yttrium $(\mathrm{Y}, Z=39)$ and zirconium $(\mathrm{Zr}$, $Z=40$ ) by about 0.2 dex. The underestimation of the observed relative abundances of the light s-process elements leads to an overestimation of the actual [hs/ls] index, which forms an indication of the neutron irradiation. In the fourth panel of Fig. 11, it can be seen that the models already overestimate this index after the second occurrence of a third dredge-up for J052043, and after the third for J053250. The overall enhancement in s-process elements, or the efficiency of the third dredge-up, on the other hand, is relatively well reproduced for both objects (see the third panel of Fig. 11) after the full series of predicted 3rd dredge-up events.

For the other two objects, J050632 and J053253, the comparison with the theoretical models is not as good. For J053253 the observed abundances are systematically too low with respect to the standard model (Fig. 11). This is another indication that the s-process and the third dredge-ups are indeed inhibited in some way (see Sect. 8).

The comparison for J050632 points to a systematic difference: the model underestimates the light s-process abundances while it heavily overestimates the observed abundance pattern for the heavy s-process elements. This results in a negative [hs/ls] index, which is not predicted by the models (see Fig. 11).

Although J050632, J052043 and J053250 are intrinsically enriched post-AGB objects with a very similar metallicity, luminosity, and hence initial mass, they display quite different levels of overabundance as well as a significantly different s-process distribution. It therefore follows that the AGB models have to be individually adapted to match the observed abundance patterns.

\section{Conclusions}

In van Aarle et al. (2011), we classified the objects J050632, J052043, J053250, and J053253 (see Table 1) as spectroscopically confirmed post-AGB stars, because of their spectral type, IR colours, and estimated luminosity. The chemical analysis we carried out in this paper was based on high-resolution UVES spectra, and confirmed their post-AGB status, as the third dredge-up clearly took place in all objects: they are C-rich, and enhanced in s-process elements. We deduced abundances of heavy s-process elements for all stars in the sample, and quantified also abundances of elements well beyond the Ba-peak. The low $\mathrm{S} / \mathrm{N}$ of the spectra in the blue spectral domain, where the lines of heavy s-process elements abondan, prevented very accurate determinations.

The metallicity of all stars except J053253 is considerably lower than the average value that is observed for the LMC. This is in accordance with the possibility that these objects are old, which means that they should have a low initial mass. The latter is also confirmed by their low luminosities. We estimate the initial mass of the three objects to be between 1 and $1.5 M_{\odot}$.

All sample stars except J053253 confirm the correlation between the efficiency of the third-dredge up and the neutron exposure that is present in the Galactic sample of post-AGB objects. The non-existence of a correlation between metallicity and neutron irradiation is also further corroborated.

The subluminosity of J053253, its small but warm infrared excess, and the deviation of the s-process abundance pattern from the expected trend (see Fig. 9) are all observational indications that this object is not evolving on a single-star evolutionary track. We argued that the dust in the system of this star may reside in a disc rather than in an freely expanding, detached shell. This would mean that this object is likely a binary. From Galactic binary post-AGB stars with a disc, it is known that the AGB evolutionary phase in these objects is truncated, because of the detected orbits (Van Winckel et al. 2009). This can explain the subluminosity and low $[\mathrm{s} / \mathrm{Fe}]$ index that are observed for this star, but radial velocity time-series are badly needed.

The $21 \mu \mathrm{m}$ feature has been detected in the spectrum of J052043 (Volk et al. 2011). As the fifteen Galactic objects that display this feature are post-carbon stars which are strongly enhanced in s-process elements (Hrivnak et al. 2008), this detection shows that this is also valid for the LMC. Similar LMC $21 \mu \mathrm{m}$ sources are known (Volk et al. 2011), and it would be useful to obtain abundances for them as well. In the SMC, there is only one known $21 \mu \mathrm{m}$ source: J004441.04-732136.4 (Volk et al. 2011), which was recently subjected to a detailed 
chemical study (De Smedt et al. 2012). This object has an initial mass of $\sim 1.3 M_{\odot}$, a metallicity of -1.3 , and turned out to be one of the most s-process enriched objects known to date. A more detailed comparison with model predictions using two different independent codes shows that very similar conflicts exist between the deduced and predicted abundances as found in this contribution.

From the comparison with AGB models, we conclude that the available AGB models overestimate the observed $\mathrm{C} / \mathrm{O}$ ratios and fail to reproduce the variety of [hs/ls] indexes that is observed in otherwise very similar stars. This seems to be a common feature (De Smedt et al. 2012). Additional near infrared spectra would be useful to determine the ${ }^{12} \mathrm{C} /{ }^{13} \mathrm{C}$ ratio of our stars, as this ratio is predicted to be very dependent on the number of dredge-up events the star has suffered on the AGB. The $\mathrm{Pb}$ production is predicted to be very high in these metal poor objects but an observational constraint will require additional high $\mathrm{S} / \mathrm{N}$ blue spectra.

This paper is the first systematic study of enriched postAGB stars in the LMC. Because the range of luminosities of this subsample is rather small, more general and systematic confrontations of the observed abundances with AGB nucleosynthetic models will only become possible when the abundances and characteristic parameters of other objects covering a wider range of metallicities and luminosities have been studied. We therefore initiated a large systematic low-resolution survey (Kamath et al. 2013) to identify the best suitable candidates both in the LMC and SMC which will enable us to quantify a wide range of chemical species in post-AGB stars covering a wide luminosity range.

Despite the small range in luminosities, the detected abundances cover a range in s-process overabundances and abundance patterns which call for very detailed, tailored models that will match the derived abundance patterns individually. Only such a systematic study can make significant progress in the understanding of the complex interplay between mixing, nucleosynthesis and mass-loss which characterise the final evolution of solar-like stars, and is the ultimate goal of our research.

Acknowledgements. E. van Aarle acknowledges support from the Fund for Scientific Research of Flanders (FWO) under grant number G.0470.07. The authors thank inspiring discussions with Tom Lloyd Evans, Lionel Siess, Stephane Goriely, Amanda Karakas, and Nadya Gorlova.

\section{References}

Abia, C., de Laverny, P., \& Wahlin, R. 2008, A\&A, 481, 161

Angelou, G. C., Church, R. P., Stancliffe, R. J., Lattanzio, J. C., \& Smith, G. H. 2011, ApJ, 728, 79

Asplund, M., Grevesse, N., Sauval, A. J., \& Scott, P. 2009, ARA\&A, 47, 481

Busso, M., Lambert, D. L., Beglio, L., et al. 1995, ApJ, 446, 775

Busso, M., Gallino, R., \& Wasserburg, G. J. 1999, ARA\&A, 37, 239

Busso, M., Palmerini, S., Maiorca, E., et al. 2010, ApJ, 717, L47

Castelli, F., \& Kurucz, R. L. 2004 [arXiv: astro-ph/0405087]

Church, R. P., Cristallo, S., Lattanzio, J. C., et al. 2009, PASA, 26, 217

Coelho, P., Barbuy, B., Meléndez, J., Schiavon, R. P., \& Castilho, B. V. 2005, A\&A, 443, 735

Condon, E. U., \& Shortley, G. 1935, The theory of atomic spectra (Cambridge University Press)
Cristallo, S., Straniero, O., Gallino, R., et al. 2009, ApJ, 696, 797

Cristallo, S., Piersanti, L., Straniero, O., et al. 2011, ApJS, 197, 17

De Smedt, K., Van Winckel, H., Karakas, A. I., et al. 2012, A\&A, 541, A67

Dekker, H., D’Odorico, S., Kaufer, A., Delabre, B., \& Kotzlowski, H. 2000, in SPIE Conf. Ser. 4008, eds. M. Iye, \& A. F. Moorwood, 534

Deroo, P., Reyniers, M., Van Winckel, H., Goriely, S., \& Siess, L. 2005, A\&A, 438, 987

Geisler, D., Grocholski, A., Sarajedini, A., Cole, A., \& Smith, V. 2009, LMC Cluster Abundances and Kinematics, eds. T. Richtler, \& S. Larsen, 133

Gielen, C., Bouwman, J., Van Winckel, H., et al. 2011, A\&A, 533, A99

Goriely, S., Hilaire, S., \& Koning, A. J. 2008, A\&A, 487, 767

Gorlova, N., Van Winckel, H., Gielen, C., et al. 2012, A\&A, 542, A27

Gray, D. F. 2008, The Observation and Analysis of Stellar Photospheres (Cambridge, UK: Cambridge University Press)

Herwig, F. 2005, ARA\&A, 43, 435

Hrivnak, B. J., Volk, K., Geballe, T. R., \& Kwok, S. 2008, in IAU Symp. 251, eds. S. Kwok, \& S. Sandford, 213

Hrivnak, B. J., Volk, K., \& Kwok, S. 2009, ApJ, 694, 1147

Kamath, D., Wood, P., Van Winckel, H., \& van Aarle, E. 2011, in 9th Pacific Rim Conference on Stellar Astrophysics, ASP Conf. Ser., 451, 187

Kamath, D., Wood, P., \& Van Winckel, H. 2013, MNRAS, submitted

Karakas, A. I. 2010, MNRAS, 403, 1413

Kovtyukh, V. V., \& Andrievsky, S. M. 1999, A\&A, 351, 597

Kupka, F., Piskunov, N., Ryabchikova, T. A., Stempels, H. C., \& Weiss, W. W. 1999, A\&AS, 138, 119

Lawler, J. E., Bonvallet, G., \& Sneden, C. 2001a, ApJ, 556, 452

Lawler, J. E., Wickliffe, M. E., den Hartog, E. A., \& Sneden, C. 2001b, ApJ, 563,1075

Lawler, J. E., Wyart, J.-F., \& Blaise, J. 2001c, ApJS, 137, 351

Lugaro, M., Karakas, A. I., Stancliffe, R. J., \& Rijs, C. 2012, ApJ, 747, 2

MacConnell, D. J., Frye, R. L., \& Upgren, A. R. 1972, AJ, 77, 384

Mashonkina, L., Gehren, T., Shi, J.-R., Korn, A. J., \& Grupp, F. 2011, A\&A, 528, A87

Pompéia, L., Hill, V., Spite, M., et al. 2008, A\&A, 480, 379

Reddy, B. E., Lambert, D. L., Gonzalez, G., \& Yong, D. 2002, ApJ, 564, 482

Rentzsch-Holm, I. 1996, A\&A, 312, 966

Reyniers, M. 2002, Ph.D. Thesis KU Leuven, Belgium

Reyniers, M., \& Van Winckel, H. 2001, A\&A, 365, 465

Reyniers, M., \& Van Winckel, H. 2003, A\&A, 408, L33

Reyniers, M., Van Winckel, H., Gallino, R., \& Straniero, O. 2004, A\&A, 417, 269

Reyniers, M., van de Steene, G. C., van Hoof, P. A. M., \& Van Winckel, H. 2007, A\&A, 471, 247

Siess, L. 2007, A\&A, 476, 893

Siess, L., Goriely, S., \& Langer, N. 2003, PASA, 20, 371

Sneden, C. A. 1973, Ph.D. Thesis, the University of Texas at Austin, USA

Sneden, C., Cowan, J. J., Lawler, J. E., et al. 2003, ApJ, 591, 936

Sneden, C., Lawler, J. E., Cowan, J. J., Ivans, I. I., \& Den Hartog, E. A. 2009, ApJS, 182, 80

Stancliffe, R. J., \& Jeffery, C. S. 2007, MNRAS, 375, 1280

Stancliffe, R. J., Dearborn, D. S. P., Lattanzio, J. C., Heap, S. A., \& Campbell, S. W. 2011, ApJ, 742, 121

Storm, J., Gieren, W., Fouqué, P., et al. 2011, A\&A, 534, A95

Sumangala Rao, S., Pandey, G., Lambert, D. L., \& Giridhar, S. 2011, ApJ, 737, L7

Takeda, Y., Taguchi, H., Yoshioka, K., et al. 2007, PASJ, 59, 1127

Tomkin, J., Lambert, D. L., Edvardsson, B., Gustafsson, B., \& Nissen, P. E. 1989, A\&A, 219, L15

van Aarle, E., van Winckel, H., Lloyd Evans, T., et al. 2011, A\&A, 530, A90

van der Marel, R. P., \& Cioni, M. 2001, AJ, 122, 1807

van der Marel, R. P., Alves, D. R., Hardy, E., \& Suntzeff, N. B. 2002, AJ, 124, 2639

Van Winckel, H. 2003, ARA\&A, 41, 391

Van Winckel, H., \& Reyniers, M. 2000, A\&A, 354, 135

Van Winckel, H., Lloyd Evans, T., Briquet, M., et al. 2009, A\&A, 505, 1221

Vassiliadis, E., \& Wood, P. R. 1994, ApJS, 92, 125

Volk, K., Hrivnak, B. J., Matsuura, M., et al. 2011, ApJ, 735, 127 